\title{
Asian-Pacific Association for the Study of the Liver (APASL) consensus guidelines on invasive and non-invasive assessment of hepatic fibrosis: a 2016 update
}

\author{
Gamal Shiha $^{1,2}$ - Alaa Ibrahim ${ }^{3} \cdot$ Ahmed Helmy $^{6} \cdot$ Shiv Kumar Sarin ${ }^{4} \cdot$ Masao Omata $^{5} \cdot$ Ashish Kumar $^{7}$. \\ David Bernstien $^{8}$ - Hitushi Maruyama9 Vivek Saraswat $^{10} \cdot$ Yogesh Chawla $^{11}$ - Saeed Hamid ${ }^{12}$ - Zaigham Abbas ${ }^{13}$. \\ Pierre Bedossa $^{14} \cdot$ Puja Sakhuja $^{15} \cdot$ Mamun Elmahatab $^{16} \cdot$ Seng Gee Lim ${ }^{17} \cdot$ Laurentius Lesmana $^{18} \cdot$ Jose Sollano $^{19}$.

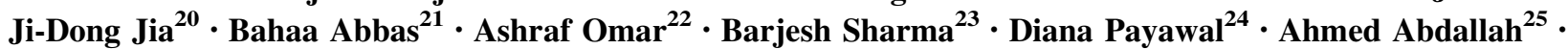 \\ Abdelhamid Serwah $^{26}$ - Abdelkhalek Hamed ${ }^{27}$ - Aly Elsayed ${ }^{28}$ - Amany AbdelMaqsod ${ }^{29}$ - Tarek Hassanein $^{30}$. \\ Ahmed Ihab $^{31} \cdot$ Hamsik GHaziuan ${ }^{32} \cdot$ Nizar Zein $^{33} \cdot$ Manoj Kumar $^{4}$
}

Received: 2 April 2016/Accepted: 13 August 2016/Published online: 6 October 2016

(C) Asian Pacific Association for the Study of the Liver 2016

\begin{abstract}
Hepatic fibrosis is a common pathway leading to liver cirrhosis, which is the end result of any injury to the liver. Accurate assessment of the degree of fibrosis is important clinically, especially when treatments aimed at reversing fibrosis are being evolved. Despite the fact that liver biopsy (LB) has been considered the "gold standard" of assessment of hepatic fibrosis, LB is not favored by patients or physicians owing to its invasiveness, limitations, sampling errors, etc. Therefore, many alternative
\end{abstract}

Gamal Shiha

g_shiha@hotmail.com

1 Internal Medicine Department, El-Mansoura Faculty of Medicine, Mansoura University, Mansoura, Egypt

2 Egyptian Liver Research Institute And Hospital (ELRIAH), Mansoura, Egypt

3 Department of Internal medicine, University of Benha, Benha, Egypt

4 Department of Hepatology, Institute of Liver and Biliary Sciences (ILBS), New Delhi, India

5 Department of Gastroenterology, University of Tokyo, Tokyo, Japan

6 Department of Tropical Medicine \& Gastroenterology, Faculty of Medicine, Assiut University, Assiut, Egypt

7 Department of Gastroenterology \& Hepatology, Ganga Ram Institute for Postgraduate Medical Education \& Research of Sir Ganga Ram Hospital, New Delhi, India

8 Division of Hepatology, North Shore University Hospital and Long Island Jewish Medical Center, New Hyde Park, New York, USA

9 Department of Gastroenterology, Chiba University Graduate School of Medicine, Chiba, Chiba Prefecture, Japan approaches to assess liver fibrosis are gaining more popularity and have assumed great importance, and many data on such approaches are being generated. The Asian Pacific Association for the Study of the Liver (APASL) set up a working party on liver fibrosis in 2007, with a mandate to develop consensus guidelines on various aspects of liver fibrosis relevant to disease patterns and clinical practice in the Asia-Pacific region. The first consensus guidelines of the APASL recommendations on hepatic fibrosis were

10 Department of Gastroenterology, Sanjay Gandhi Postgraduate Institute of Medical Sciences, Lucknow, Uttar Pradesh, India

11 Post Graduate Institute of Medial Education \& Research, Chandigarh, India

12 Department of Medicine, The Aga Khan University \& Hospital, Stadium Road, Karachi, Pakistan

13 Department of Hepatogastroenterology, Sindh Institute of Urology and Transplantation, Karachi, Pakistan

14 Department of Pathology, Physiology and Imaging, University Paris Diderot, Paris, France

15 Govind Ballabh Pant Hospital, Maulana Azad Medical College, New Delhi, India

16 Department of Hepatology, Bangabandhu Sheikh Mujib Medical University, Dhaka, Bangladesh

17 Department of Medicine, Yong Loo Lin School of Medicine, National University of Singapore, Singapore, Singapore

18 Department of Medicine, University of Indonesia, Depok, Indonesia

19 University of Santo Tomas, España Blvd, Manila, Philippines

20 Liver Research Centre at the Beijing Friendship Hospital, Capital University in Beijing, Beijing, China 
published in 2009. Due to advances in the field, we present herein the APASL 2016 updated version on invasive and non-invasive assessment of hepatic fibrosis. The process for the development of these consensus guidelines involved review of all available published literature by a core group of experts who subsequently proposed consensus statements followed by discussion of the contentious issues and unanimous approval of the consensus statements. The Oxford System of the evidence-based approach was adopted for developing the consensus statements using the level of evidence from one (highest) to five (lowest) and grade of recommendation from A (strongest) to D (weakest). The topics covered in the guidelines include invasive methods (LB and hepatic venous pressure gradient measurements), blood tests, conventional radiological methods, elastography techniques and cost-effectiveness of hepatic fibrosis assessment methods, in addition to fibrosis assessment in special and rare situations.

Keywords Liver fibrosis · Invasive assessment, noninvasive assessment - Cirrhosis - Hepatitis C - Hepatitis B . Chronic liver disease $\cdot$ Non-alcoholic steatohepatitis · Graft fibrosis

\begin{tabular}{|c|c|c|}
\hline \multicolumn{3}{|c|}{ Abbreviations } \\
\hline \multicolumn{2}{|c|}{ AASLD } & $\begin{array}{l}\text { American Association for the Study of Liver } \\
\text { Diseases }\end{array}$ \\
\hline \multicolumn{2}{|c|}{$\mathrm{ADC}$} & Apparent diffusion \\
\hline \multicolumn{2}{|c|}{ APASL } & Asian Pacific Association for the Study of Liver \\
\hline \multicolumn{2}{|c|}{ APRI } & AST platelet ratio index \\
\hline \multicolumn{2}{|c|}{ aPTT } & Activated partial thromboplastin time \\
\hline \multicolumn{2}{|c|}{ ARFI } & Acoustic radiation force impulse \\
\hline \multicolumn{2}{|c|}{ AUROC } & Area under the receiver-operator characteristic \\
\hline \multicolumn{2}{|c|}{ BIPED } & $\begin{array}{l}\text { Burden of disease, investigative, prognostic, } \\
\text { efficacy of intervention and diagnostic }\end{array}$ \\
\hline \multicolumn{2}{|c|}{ CDS } & Cirrhosis discriminant score \\
\hline \multicolumn{3}{|c|}{$\begin{array}{l}\text { Department of Internal Medicine, Military Medical } \\
\text { Academy, Cairo, Egypt }\end{array}$} \\
\hline 22 & \multicolumn{2}{|c|}{$\begin{array}{l}\text { Tropical Medicine Department, Cairo Medical School, Cairo, } \\
\text { Egypt }\end{array}$} \\
\hline 23 & \multicolumn{2}{|c|}{$\begin{array}{l}\text { Department of Gastroenterology, GB Pant Hospital, } \\
\text { New Delhi, India }\end{array}$} \\
\hline 24 & \multicolumn{2}{|c|}{$\begin{array}{l}\text { Section of Gastroenterology, Cardinal Santos Medical } \\
\text { Center, San Juan City, Metro Manila, Philippines }\end{array}$} \\
\hline 25 & \multicolumn{2}{|c|}{ Pediatric Hospital, Mansoura University, Mansoura, Egypt } \\
\hline 26 & \multicolumn{2}{|c|}{$\begin{array}{l}\text { College of Medicine, Taif University, Taif, Kingdom of } \\
\text { Saudi Arabia }\end{array}$} \\
\hline 27 & \multicolumn{2}{|c|}{$\begin{array}{l}\text { Hepatology and Diabetes Unit, Military Medical Academy, } \\
\text { Cairo, Egypt }\end{array}$} \\
\hline 28 & \multicolumn{2}{|c|}{$\begin{array}{l}\text { Hepatology \& GIT Department, AHF Center Abu Dhabi, } \\
\text { Abu Dhabi, United Arab Emirates }\end{array}$} \\
\hline
\end{tabular}

\section{Abbreviations}

APASL Asian Pacific Association for the Study of Liver APRI AST platelet ratio index

Activated partial thromboplastin time

AUROC Area under the receiver-operator characteristic

BIPED Burden of disease, investigative, prognostic, efficacy of intervention and diagnostic CDS Cirrhosis discriminant score

\begin{tabular}{|c|c|}
\hline CDUS & Color Doppler US \\
\hline CEUS & Contrast-enhanced ultrasound \\
\hline $\mathrm{CHC}$ & Chronic hepatitis \\
\hline $\mathrm{CI}$ & Confidence interval \\
\hline CPA & Collagen proportionate area \\
\hline $\mathrm{C} / \mathrm{RL}$ & Caudate lobe width to the right lobe width \\
\hline $\mathrm{CT}$ & Computed tomography \\
\hline CPTs & Child-Pugh-Turcotte score \\
\hline DWMRI & $\begin{array}{l}\text { Diffusion-weighted magnetic resonance } \\
\text { imaging }\end{array}$ \\
\hline $\mathrm{ECM}$ & Extracellular matrix \\
\hline ELF & Enhanced liver fibrosis \\
\hline FHVP & Free hepatic venous pressure \\
\hline Fib-4 & Fibrosis 4 \\
\hline FT & Fibrotest \\
\hline GRADE & $\begin{array}{l}\text { Grading of recommendations assessment } \\
\text { development and evaluation }\end{array}$ \\
\hline HA & Hyaluronic acid \\
\hline HAI & Histological activity index \\
\hline HAP & Hepatic arterial phase \\
\hline $\mathrm{HCC}$ & Hepatocellular carcinoma \\
\hline HBV & Hepatitis B virus \\
\hline $\mathrm{HCV}$ & Hepatitis $\mathrm{C}$ virus \\
\hline HSCs & Hepatic stellate cells \\
\hline HVPG & Hepatic venous pressure gradient \\
\hline INR & International normalized ratio \\
\hline $\mathrm{kPa}$ & Kilopascals \\
\hline LB & Liver biopsy \\
\hline LR & Likelihood ratio \\
\hline LSM & Liver stiffness measurement \\
\hline MELD & Model for end-stage liver disease \\
\hline $\operatorname{miR}-29$ & Micro-RNA-29 \\
\hline MMPs & Matrix metalloproteinase \\
\hline MRE & Magnetic resonance elastography \\
\hline MRI & Magnetic resonance imaging \\
\hline MRS & Magnetic resonance spectroscopy \\
\hline
\end{tabular}

29 Internal Medicine Department, Faculty of Medicine Cairo University, Liver Transplant Unit Manial Hospital and Liver ICU French Hospital, Cairo University, Cairo, Egypt

30 Chula Vista Medical Center, Chula Vista, CA, USA

31 Molecular Pathology Unit \& Research Group, German University in Cairo, Cairo, Egypt

32 Department of Hepatology, Nork Clinical Hospital of Infectious Diseases, Yerevan, Armenia

33 Department of Gastroenterology and Hepatology, Cleveland Clinic, Cleveland, USA 
NAFLD Non-alcoholic fatty liver disease

NASH Non-alcoholic steatohepatitis

NOTES Natural orifice transluminal endoscopic surgery

PDUS Power Doppler ultrasound

PIIINP Amino-terminal propeptide of procollagen type III

PSC Primary sclerosing cholangitis

PT Prothrombin time

PVP Portal venous phase

RFP Rapid fibrosis progression

RN Regenerating nodules

ROIs Regions of interest

RR Relative risk

SH-PPF Schistosomal hepatic periportal fibrosis

SIR Society of interventional radiology

SNR Signal-to-noise ratio

SPIOs Superparamagnetic iron

SSI Supersonic shear imaging

SWE Shear wave elastography

TE Transient elastography

TEG Thromboelastography

TIMP Tissue inhibitors of metalloproteinases

TJLB Transjugular liver biopsy

TPO Thrombopiotein

US Ultrasound

WHVP Wedged hepatic venous pressure

\section{Introduction}

The degree of liver fibrosis is one of the most important diagnostic and prognostic assessments in chronic liver disease. Clinical manifestations of liver disease and liver dysfunction accompany architectural changes of the liver parenchyma that are a result of advanced stages of liver fibrosis. Globally, among the etiologies of hepatic fibrosis, viral infections (chronic hepatitis B and C) and alcoholinduced liver damage are the most common. Also the rising obesity rates worldwide have increased the risk of liver injury because of nonalcoholic fatty liver disease (NAFLD) and nonalcoholic steatohepatitis (NASH). Other etiologies of chronic liver injury include drug-induced toxicity, other liver infections (e.g., schistosomiasis), immune-mediated liver diseases (e.g., autoimmune hepatitis), metabolic disorders (e.g., lipid, glycogen or metal storage disorders) and cholestatic liver diseases.

Often regarded as the gold standard for fibrosis assessment, liver biopsy (LB) is invasive in nature with associated risks. Moreover, LB has its own limitations owing to inter- and intra-observer variability and the small amount of tissue that is typically obtained with this procedure. Advances in the development of serologic tests and conventional and newer imaging techniques have been shown to reduce the need for LB for diagnosing hepatic fibrosis. Once cirrhosis develops, complications may develop including ascites, portal hypertension, encephalopathy, liver failure and an increased risk of hepatocellular carcinoma (HCC). At present, the only curative treatment for end-stage cirrhosis is liver transplantation. Previously, it was thought that liver fibrosis and end-stage liver disease (cirrhosis) were irreversible. Recent work suggests that liver fibrosis may be modified by treatment. Thus, new medical therapies to decrease or halt the progression of fibrosis or reverse the fibrosis are urgently needed. The discovery of the cells involved in liver fibrosis and evidence that fibrosis is a dynamic and even reversible process have led to continuous research in uncovering of the molecular mechanisms of fibrosis in order to discover new therapeutic strategies. Several advances have and are being made in this area including cell isolation and culture techniques for hepatic stellate cells (HSCs) from rodents and humans; the development of animal models of liver injury due to hepatic toxins (e.g., carbon tetrachloride, thioacetamide, dimethylnitrosamine), bile duct ligation and/or immune injury; genetic models of liver disease and micro-RNA-29 (miRNA-29) in human and murine liver fibrosis. As these pathways of fibrogenesis are further clarified, the opportunity to develop new diagnostic tools and therapeutic strategies will accelerate.

\section{Methodology of the guideline development}

These APASL clinical practice guidelines represent an update of the last APASL guidelines published in 2009 [1]. The 2015 guidelines were developed by a panel of experts' chosen by the APASL Governing Board, peer-reviewed by the experts and approved by the APASL Governing Board. The clinical practice guidelines are based on evidence from existing publications, and, if evidence was unavailable, on the experts' personal experience and opinion. Manuscripts and abstracts of important meetings published till March 2014 have been evaluated. The evidence and recommendations in these guidelines have been graded according to the Grading of Recommendations Assessment Development and Evaluation (GRADE) system [2, 3] (Table 1).

\section{Invasive and non-invasive markers for the diagnosis of liver fibrosis}

Investigations and "routine" scanning methods are unable to determine whether cirrhosis or fibrosis is present [4]. The gold standard to diagnosis liver fibrosis or cirrhosis has been LB, but this has been associated with significant morbidity even though mortality rates are very low. The 
Table 1 Grading of evidence and recommendations (adapted from the GRADE system) [2]

\begin{tabular}{ll}
\hline & Notes \\
\hline $\begin{array}{l}\text { Grading of evidence } \\
\text { High quality } \\
\text { Moderate quality }\end{array}$ & $\begin{array}{l}\text { Further research is very unlikely to change our confidence in the estimate of effect } \\
\text { Further research is likely to have an important impact on our confidence in the estimate } \\
\text { of effect and may change the estimate }\end{array}$ \\
Fow or very low quality & $\begin{array}{l}\text { Further research is very likely to have an important impact on our confidence in the } \\
\text { estimate of effect and is likely to change the estimate. Any estimate of effect is } \\
\text { uncertain }\end{array}$ \\
$\begin{array}{l}\text { Factors influencing the strength of the recommendation included the quality of the } \\
\text { evidence, presumed patient-important outcomes and cost } \\
\text { Variability in preferences and values, or more uncertainty: more likely a weak } \\
\text { recommendation is warranted. Recommendation is made with less certainty; higher } \\
\text { cost or resource consumption }\end{array}$ \\
\hline
\end{tabular}

invasive nature makes it unacceptable to many patients; consequently, non-invasive methods have evolved over the last decade. Progress in understanding of the mechanisms of fibrosis has led to the identification of many potential markers of fibrosis, which appear capable of identifying early and advanced hepatic fibrosis. Standard cross-sectional imaging studies will only identify or exclude advanced fibrosis. Novel technologies such as transient elastography (TE) and magnetic resonance elastography (MRE) show promise as non-invasive methods of testing for hepatic fibrosis.

\section{Methodologies and statistical considerations}

The best discriminants of non-invasive tests of liver fibrosis are those tests that show a prognostic link and are independently validated [5]. This strategy, however, has not been widely accepted as yet. Any non-invasive marker that quantitatively estimates liver fibrosis needs evaluation of its diagnostic accuracy, which is done using the LRs and the area under the receiver-operator characteristic (AUROC) curve. AUROC combines the sensitivity and specificity of a given quantitative marker for the diagnosis of a specific definition of fibrosis. Sensitivity is usually assessed in patients with advanced fibrosis (i.e., stages F2, F3 and F4 in the METAVIR scoring system) and specificity in non-advanced fibrosis (i.e., stages F0, F1). Any new method of assessing liver fibrosis needs to be compared with LB. Relatively high misclassification rates of the gold standard, i.e., the LB, make it impossible to achieve a perfect concordance (AUROC $\sim 1$ ). When errors in a diagnostic test and gold standard are independent, the observed sensitivity and specificity of the diagnostic test are underestimated. When examining diagnostic studies, we need to be cognizant of the measures being evaluated and their significance. The typical test spectrum includes sensitivity and specificity, which can guide decisions on which test to select, while positive and negative predictive values are aimed to rule in or rule out disease. This is affected by the prevalence of the disease in question and hence cannot be broadly applied. To overcome this problem, likelihood ratios (LRs) and AUROC curve graphs have been utilized. AUROC graphs may not be able to discriminate different tests when the differences are relatively minor. Hence, the LRs may be better able to determine a difference [6]. A proposed method was to utilize a clinical utility model in which predetermined thresholds for sensitivity and specificity are chosen and the test performance classified as correct, incorrect or indeterminate [6]. While this is a potentially practical method, there is not yet agreement on what these thresholds would be. In addition, performance of diagnostic tests may vary according to the severity and clinical presentation of the disease, called spectrum bias [7]. One method of reducing spectrum bias is to use a statistical correction to account for the differences in prevalence of severe fibrosis within the study population called dynamic actor network analysis (DANA) [8]. This is an adjusted AUROC $=$ observed AUROC + [0.1056 (2.5observed DANA)] to account for the differences in prevalence of severe fibrosis in the population.

Based on the limitations of LB, a large variety of noninvasive investigations have been tested to evaluate their diagnostic potential in liver fibrosis. These can broadly be divided into blood biomarkers, imaging methods and elastography methods. An alternative method has been to use an algorithm-based approach.

\section{LB for assessment of liver fibrosis}

LB serves as an important tool for diagnosis, prognosis and treatment of liver diseases. In 1958, Menghini [9] published the first description of LB as it pertained to the study of fibrosis. Since then LB has been considered to be the 
procedure of choice to assess the amount of fibrosis in the tissue. LB techniques over the years have undergone many improvements and changes, and it is still considered to be the gold standard, even in this era of serologic markers, better imaging techniques and advanced molecular techniques for diagnosis and quantification of hepatitis viruses [10].

\section{Consensus statement}

- LB is considered as the gold standard for diagnosing liver fibrosis, but sampling errors and both intra- and inter-observer agreement on biopsy samples may lead to poor reproducibility for many liver biopsies, and the procedure is invasive and expensive (A1).

\section{Techniques of $\mathrm{LB}$}

Several techniques may be used to obtain liver tissue. While percutaneous LB remains the most common practice, LB can also be performed via the transvenous route, during surgery or by laparoscopy, especially in patients with contraindications to a percutaneous approach.

\section{Percutaneous LB}

\section{Preparation for percutaneous $L B$}

Prior to performance of LB, patients should be educated about their liver disease and about the biopsy procedure (including alternatives to LB, risks, benefits and limitations of LB). A complete history and physical examination, a review of medications and measurement of clotting parameters should be done prior to the procedure. A light meal $2-4 \mathrm{~h}$ before the procedure may facilitate gallbladder emptying and reduce the risk of gallbladder puncture and also help avoid a vasovagal response during or shortly after the procedure. Percutaneous LB has traditionally been avoided in patients with a prothrombin time $(\mathrm{PT})>4$ s over control, an international normalized ratio (INR) $>1.5$ or a platelet counts $<60,000 / \mu l$. Patients with the above derangements should undergo transjugular liver biopsy (TJLB) and receive fresh frozen plasma and/or platelet transfusions before the procedure.

Few data are available with which to guide management about the timing of discontinuation of anticoagulants and/ or antiplatelet agents prior to LB. A list of different drugs and their recommended stopping time is shown in Table 2. Anticoagulation should be resumed when safely possible; in the absence of immediate bleeding or adverse events, unfractionated heparin should be reinitiated $2-6 \mathrm{~h}$ after LB . Timing of restarting anticoagulants is greatly debated.
However, since delayed bleeding has been reported in patients on oral anticoagulants, oral anticoagulation should not be restarted immediately, but can be resumed $48-72 \mathrm{~h}$ after the biopsy [11].

Management of specific drugs should be handled on a case-by-case basis, and in all patients, the risk of discontinuing these medications must be weighed against the (potential) risk of bleeding during/after LB. Written consent documenting the discussion about the procedure and any potential complications, with the patient and/or a proxy, should be obtained.

\section{Technique and process of percutaneous $L B$}

If patients are feeling anxious, intravenous fentanyl and midazolam can alleviate apprehension, facilitate the procedure, provide some post-procedure relief of pain and achieve some degree of amnesia.

\section{Use of imaging for percutaneous $L B$}

Ultrasound (US) can be used to either "mark" the biopsy site or the US can be used in real time by the operator. Image guidance for LB (either marking or in real time) should be considered in patients with known specific mass lesions and in those with previous intra-abdominal surgery who may have adhesions, allowing avoidance of other structures in the latter situation. It may be especially useful in specific situations, including: (1) patients with small livers that are difficult to percuss, (2) obese patients or (3) patients with clinically demonstrable ascites. Use of ultrasonography has resulted in better outcomes, is cost effective and already widely utilized; however, it is not obligatory [12].

\section{Devices used for percutaneous $L B$}

Needles used to perform percutaneous LB are broadly categorized as aspiration needles (Menghini, Klatskin and Jamshidi needles) and cutting needles (Vim Silverman and 'Tru-Cut' needles and spring-loaded needles with triggering mechanisms). Comparisons of Menghini aspiration and 'Tru-Cut' needles have shown increased rates of tissue sample fragmentation with the aspiration needles compared with the cutting-type needles, especially in the presence of cirrhosis $[13,14]$. Tru-Cut biopsies are significantly longer (12 versus $8 \mathrm{~mm} ; p<0.001$ ) and contain a greater number of portal tracts $(16$ versus $6 ; p<0.001)$ than aspirationtype needles [13]. Among the cutting type devices, it has been reported that the automated needles acquire slightly longer tissue samples (1.7 versus $1.5 \mathrm{~mm} ; p=0.05$ ) [15]. Non-automated cutting needles remain in the liver for a modestly longer time during the biopsy and may pose 
Table 2 Management of antithrombotic medications prior to liver biopsy

\begin{tabular}{|c|c|c|}
\hline Drug class & Agents & Suggested management \\
\hline \multirow[t]{2}{*}{$\begin{array}{l}\text { Antiplatelet } \\
\text { agents }\end{array}$} & $\begin{array}{l}\text { Aspirin, } \\
\text { dipyramidole, } \\
\text { NSAIDS }\end{array}$ & Hold for 7-10 days \\
\hline & $\begin{array}{l}\text { Thienopyridines } \\
\text { (e.g., clopidogrel, } \\
\text { ticlopidine) }\end{array}$ & Hold for 7-10 days \\
\hline \multirow[t]{6}{*}{ Anticoagulants } & Warfarin & Hold for 5 days \\
\hline & $\begin{array}{l}\text { Unfractionated } \\
\text { heparin }\end{array}$ & Hold for 4-6 h \\
\hline & $\begin{array}{l}\text { Low molecular } \\
\text { weight heparin }\end{array}$ & Hold for $12-24 \mathrm{~h}$ \\
\hline & Dabigatran & $\begin{array}{l}\text { Hold for } 1-2 \text { days if the } \\
\text { estimated } \mathrm{Cl}_{\mathrm{cr}} \geq 50 \mathrm{ml} / \\
\text { min, or } 3-5 \text { days if } \mathrm{Cl}_{\mathrm{cr}} \text { is } \\
<50 \mathrm{ml} / \mathrm{min}\end{array}$ \\
\hline & Rivaroxaban & Hold for $24 \mathrm{~h}$ \\
\hline & Fondaparinux & $\begin{array}{l}\text { Hold for } 2-4 \text { days in patients } \\
\text { with normal renal function. } \\
\text { Hold longer in the setting } \\
\text { of renal insufficiency }\end{array}$ \\
\hline
\end{tabular}

NSAIDs nonsteroidal anti-inflammatory drugs. $\mathrm{Cl}_{c r}$, creatinine clearance

Table 3 Contraindications to percutaneous liver biopsy

Uncooperative patient
Bleeding tendency: prothrombin time $(\mathrm{PT}) \geq 4$ s over control or
international normalized ratio (INR) $>1.5$ platelet count
$\quad<60,000 / \mathrm{mm}^{3}$
Inability to transfuse blood products
Serious consideration of echinococcal disease
Presumed hemangioma or other vascular tumors
Ascites
Infection in right pleural cavity and/or below right diaphragm

additional bleeding risk because of this added time [16]. Also, larger bore needles may have a higher risk of bleeding [17].

\section{Plugged biopsy}

The plugged biopsy has been proposed as being potentially safer than standard percutaneous biopsy among certain patients (i.e., those believed to be at high risk for bleeding such as those with coagulopathy and/or thrombocytopenia or a small cirrhotic liver). The plugged biopsy is a modification of the percutaneous method in which the biopsy track is plugged with collagen or thrombin (or other materials) as the cutting needle is removed from a sheath while the breath is still being held [18]. Plugged percutaneous biopsy may provide an alternative in those with mild coagulation abnormalities and ascites.

\section{Post-procedural care}

After the successful completion of the biopsy procedure, the patient should be observed for a period of time with vital signs being taken every $15 \mathrm{~min}$ for the first hour and then every $30 \mathrm{~min}$ for the second hour. Conventionally, the patient should remain in the right lateral decubitus position during this time. The majority of complications occurred within $1 \mathrm{~h}$ during the observation period or within $24 \mathrm{~h}$ after discharge. Thus, an observation time of 2-4 h after ambulatory percutaneous LB is safe after which patients may be discharged with detailed instructions on when to seek medical help in case worrying symptoms develop.

\section{Contraindications of percutaneous LB (Table 3)}

Uncooperative patients Lack of patient's cooperation may increase the risk of complications. Uncooperative patients needing LB should get biopsies via the transvenous routes using sedation.

Ascites Whether the risk of bleeding in patients with ascites is increased is unclear; those with moderate ascites may be able to undergo a therapeutic paracentesis prior to biopsy, but in most cases a transjugular approach is used since many of these patients will have coexisting coagulation impairment.

Mass lesions Biopsy of most liver mass lesions (typically by radiology experts using a real-time imaging technique) is safe. In general, fine-needle aspiration provides cytology, which typically has less diagnostic value than an adequate core biopsy. Biopsy of known vascular lesions should generally be avoided, although image-guided biopsy of potential vascular lesions may be safe as a needle path can be selected whereby normal (or non-tumorous) parenchyma can be interposed between the liver capsule and the lesion. When US with color Doppler is used to guide the biopsy, larger tumor and liver vessels can also be identified and avoided. Biopsy of malignant lesions is associated with a risk of tumor spread usually along the biopsy track, which is very low, from 0 to $0.13 \%$ in recent series [19, 20]. Biopsy and/or aspiration of infectious lesions is generally safe. Traditionally an echinococcal cyst (hydatid disease) represents an absolute contraindication to LB because piercing of the cyst may be associated with fatal anaphylaxis. However, available data suggest that careful aspiration of these lesions with 19- to 22-gauge needles is relatively safe [21]. Nevertheless, if suspected, consideration and preparation for possible anaphylaxis are warranted. 
Impaired hemostasis There is no definitive correlation between risk of bleeding and level of coagulopathy [22]. The lack of reproducibility of conventional INR among different laboratories is inconsistent with having a set cutoff for this number [23]. The commonly used prothrombin time-intenational normalization ratio (PT-INR) is derived from coumadin-treated reference ranges and thus lacks applicability to liver disease where there are abnormalities in both the procoagulant and anticoagulant system [24]. An abnormal increase in the PT-INR often correlates with an increased risk of bleeding, and correcting the abnormal PT-INR with plasma replacement therapy or recombinant activated factor VII will reduce that risk. However, the available data do not appear to support these assumptions, particularly in patients with mild coagulopathy defined as an INR of $<2.0$ [25]. Another measure of coagulation in liver disease has been introduced, the "INRLIVER;" it recalculates the international sensitivity index from a reference point of patients with liver disease rather than coumadin-treated patients as has been the convention [26]. Whether this test will provide a reliable measure of bleeding risk remains to be determined. Thromboelastography (TEG) assesses global hemostasis involving both plasma and cellular components. TEG parameters were a better predictor of bleeding risk in posttransplant patients who underwent renal biopsy in comparison to conventional tests [26]. SONOCLOT is a method that assesses in vitro global coagulation, especially systemic fibrinolysis, is an efficient tool to assess coagulopathies in patients with liver disease and can predict hypercoagulability [27]. Studies to validate the superiority of global hemostatic tests such as TEG and SONOCLT over PT/INR, aPTT and platelet counts prior to LB are unmet needs.

In Japan, Eltrombopag is not covered by health insurance when used for patients with chronic hepatitis complicated with thrombocytopenia scheduled for invasive techniques such as LB. Lusutrombopag, an orally bioavailable thrombopoietin receptor agonist developed for patients with thrombocytopenia prior to elective invasive procedure, is covered for the same indication [28]. These medications are not available in many countries and have not been adequately investigated.

Several conditions are more definitively associated with enhanced risk of bleeding and therefore warrant additional caution. These include patients with factor VIII (FVIII) or IX (FIX) deficiency, von-Willebrand's disease and other hereditary bleeding disorders, and those with sickle cell anemia. Patients with known underlying coagulopathy requiring LB represent a challenge, but it should be emphasized that LB (percutaneous or transvenous) can be performed in these patients (with definitive factor replacement).

\section{Complications of percutaneous LB}

Complications resulting from LB range from pain, which is common, to more serious events such as bleeding, perforation of a neighboring organ, infection or death, all of which are uncommon (Table 4). Sixty percent of complications are recognized within $2 \mathrm{~h}$ after the procedure and $96 \%$ within $24 \mathrm{~h}$ [29]. Fatal complications typically occur within $6 \mathrm{~h}$. A hospitalization rate of 1.4-3.2\% for the management of complications following LB has been reported, with pain or hypotension being the predominant cause [30]. Mortality after LB has been reported to vary from 0.0088 to $0.3 \%$ [31].

\section{Consensus statements}

- Patients should be informed about their liver disease and about LB including the alternatives, its benefits, risks and limitations (A1).

- Written consent documenting the discussion about the procedure and any potential complications, with the patient and/or a proxy should be obtained (A1).

- Four hours fasting before the procedure may be considered (C2).

- Anticoagulants should be discontinued prior to LB. Warfarin should generally be discontinued at least 5 days prior to LB. Warfarin may be restarted $48-72 \mathrm{~h}$ after LB (B2).

- Antiplatelet medications and non-steroidal anti-inflammatory drugs should be discontinued $\sim 7-10$ days before, although there is uncertainty surrounding the need for their discontinuation. These may be restarted 48-72 $\mathrm{h}$ after the procedure (B2).

- In all patients, the risk of discontinuing anticoagulants must be weighed against the (potential) risk of bleeding during/after LB (A1).

- US guidance is preferred, though not mandatory, because it is likely to reduce the risk of complications from LB (A1).

- US guidance is preferred, though not mandatory, because it is likely to reduce the risk of complications from LB (A1).

- Image-guided LB is recommended in certain clinical situations including in patients with known intrahepatic lesions (real-time imaging is preferred); with previous intraabdominal surgery who may have adhesions; patients with small livers that are difficult to percuss; obese patients and patients with clinically evident ascites (A1).

- Vital signs must be frequently monitored (at least every 15 min for the first hour) after LB (B1). 
Table 4 Complications of percutaneous liver biopsy

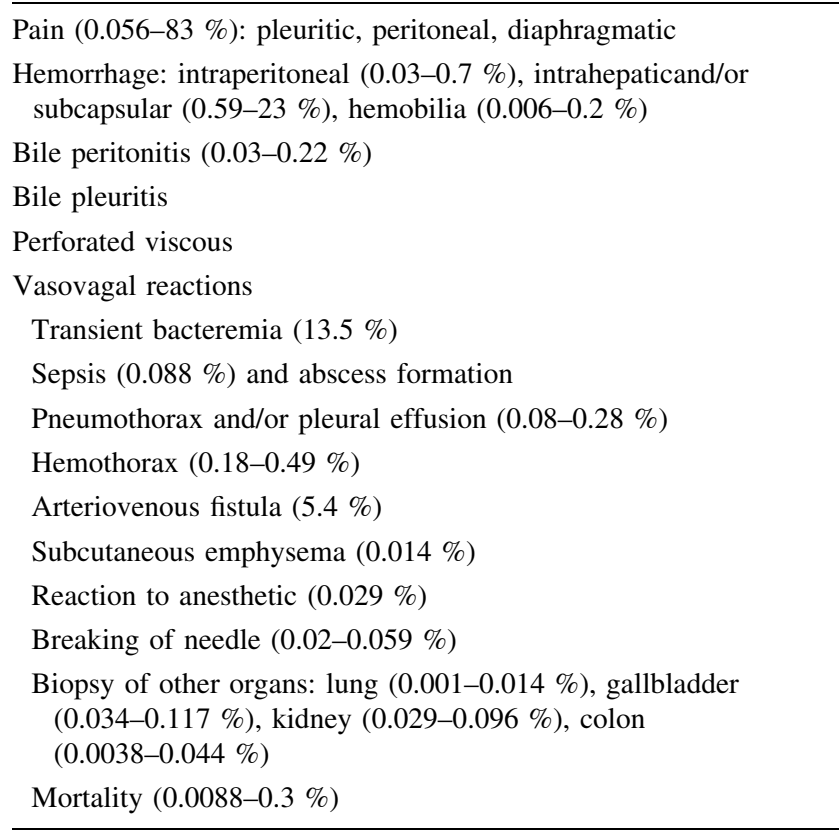

- Percutaneous LB can be performed as a daycare procedure; an observation time of $2-4 \mathrm{~h}$ after the biopsy is safe (B2).

- Percutaneous LB with or without image guidance should not be utilized in uncooperative patients. Uncooperative patients who require LB should undergo the procedure under general anesthesia or via the transvenous route (A1).

- In patients with clinically evident ascites requiring LB, a transvenous approach is recommended, though percutaneous biopsy (after removal of ascites) is acceptable (A1).

- In general, prothrombin time $(\mathrm{PT}) \geq 4$ s over control or international normalized ratio (INR) $>1.5$ or platelet count $<60,000 / \mathrm{mm}^{3}$ is considered a contraindication to percutaneous LB (B2).

- Platelet transfusion should be considered soon before LB when platelet levels are $<60,000 / \mathrm{ml}$ (C2).

- PT, INR, aPTT and platelet counts do not assess hyperfibrinolysis and thrombocyte dysfunction as an event that increases bleeding risk in liver disease patients; hence, research to assess the role of LIVER INR or global hemostatic tests such as TEG and SONOCLOT prior to LB is needed (C2).

\section{Transjugular liver biopsy (TJLB)}

TJLB provides an alternative mechanism for obtaining liver tissue in patients with contraindications to percutaneous biopsy (Table 5). The transjugular approach offers
Table 5 Indications for transjugular liver biopsy (TJLB)

Ascites
Coagulopathy
Thrombocytopenia
Fulminant hepatic failure
Obesity
Postoperative liver transplant
Assessment of hepatic venous pressure gradient (HVPG)
Refusal or failure of percutaneous biopsy

the added benefit of being able to obtain a hepatic venous pressure gradient, a parameter that is increasingly recognized as an important clinical and prognostic marker.

\section{Indications of TJLB}

Significant ascites and bleeding tendency are the most common indications for TJLB [32]. TJLB has been shown to have a diagnostic and prognostic role in those with acute hepatic failure [33]. In those found to have submassive or massive necrosis, prognosis was poor. TJLB has also been reported to be safe and helpful in the early postoperative period after liver transplant because of coagulopathy or ascites [34]. Other common indications for TJLB are obesity and failure of percutaneous biopsy.

\section{Technique of TJLB}

TJLB is performed under fluoroscopic guidance. Modest amounts of conscious sedation can be used. TJLB consists of obtaining liver tissue through a rigid cannula introduced into one of the hepatic veins, typically using jugular venous access. The total procedure time is usually $30-60 \mathrm{~min}$. The technical success rate for a TJLB procedure ranges from 87 to $97 \%$.

\section{Contraindications to TJLB}

There are no specific contraindications to performing a TJLB. Abnormal coagulation is not a contraindication, but attempts should be made to correct coagulation derangements before proceeding. The Society of Interventional Radiology (SIR) guidelines establish that additional blood products should be administered if the INR is $>2.5$ times the control and the platelet count is $<50,000$ [35]. Other relative contraindications to TJLB include lack of suitable venous access and biopsy of a focal lesion. In some cases, a combination of transjugular and US-guided techniques may be used to sample focal lesions in patients who are at high risk of bleeding and have the lesion in the vicinity of one of the hepatic veins. 


\section{Complications of TJLB}

The complication rates are low and range from 1.3 to $6.5 \%$ [32]. The majority of complications are minor and related to bleeding at the puncture site or abdominal pain related to the presence of a small hematoma distending the liver capsule. Major complications have been reported in $0.6 \%$ of patients [32]. The reported mortality rate is $<0.1 \%$ for adults and $0.1 \%$ for children. Mortality may be related to hemorrhage from an extracapsular liver puncture, capsular perforation after a wedge injection, perforation of the hepatic artery or ventricular dysrhythmia. Other complications include hemobilia and pseudoaneurysm. In general, reports in the literature agree that complication rates are lower during TJLB when compared with the percutaneous or mini-laparoscopy approach [36-39].

\section{Consensus statements}

- TJLB is indicated for patients with diffuse liver disease who need a biopsy and have either a contraindication to percutaneous biopsy or require hemodynamic evaluation as part of their diagnostic workup (A1).

\section{Surgical or laparoscopic liver biopsy}

In many circumstances, a surgical or laparoscopic approach is utilized because the liver is noted to be abnormal in appearance prior to planned surgery or at the time of surgery. Biopsy in this situation is performed either with typical needle devices or by wedge resection. Wedge resection may produce overestimates of fibrosis due to its proximity to the capsule. Laparoscopic LB allows adequate tissue sampling under direct vision, with direct (and immediate) control of bleeding. Those with special expertise generally perform it, typically under general anesthesia. Most studies that have compared laparoscopic biopsy to transthoracic percutaneous biopsy have demonstrated greater accuracy in diagnosing cirrhosis with the former approach, probably because of the added benefit of peritoneal inspection [40]. Complications of this method include general anesthesia, local abdominal wall or intraperitoneal trauma, and bleeding. Expense and the requirement for special expertise have limited its use. New laparoscopic techniques may facilitate laparoscopic LB and could theoretically be performed safely at low cost. An exciting possibility is that techniques extending from natural orifice transluminal endoscopic surgery (NOTES) could be used to perform LB [41].

\section{Consensus statement}

- In certain special circumstances needle biopsy or wedge biopsy can be performed during surgical or laparoscopic procedures. Only those with special expertise should perform this procedure (B2).

\section{Limitations of LB and adequacy of the specimen's size}

Besides its invasive nature and associated complications, LB has many problems including the following: the specimen obtained represents only $1 / 50,000$ of the total liver volume; problems related to the specimen obtained in terms of its size; biopsies less than $3 \mathrm{~cm}$ in length of fragmented biopsies leads to reduction in sensitivity for detection of fibrosis.

\section{What is an adequate size of $L B$ specimen for fibrosis evaluation?}

The adequate size of LB specimen is often a matter of much speculation, as this has to balance the adequacy of the information provided by the biopsy with the procedure presenting a risk to the patient. Even a tiny biopsy providing the relevant information (such as the focus of malignancy) may be perceived as adequate. However, in the context of the grading and staging of liver biopsies for chronic viral hepatitis, the definition of adequacy is required. The Royal College of Pathologists has defined adequacy of liver core biopsies for medical diseases as follows [42]:

- Inadequate-less than $10 \mathrm{~mm}$ length and/or less than six portal tracts;

- Compromised-at least $10 \mathrm{~mm}$ length and 6 portal tracts, but less than $20 \mathrm{~mm}$ and 11 portal tracts;

- Adequate-at least $20 \mathrm{~mm}$ length and 11 portal tracts.

Virtual biopsies were used to define adequacy and showed that a biopsy length of at least $25 \mathrm{~mm}$ is necessary to evaluate fibrosis accurately with a semiquantitative score [43]. No substantial benefit is obtained for longer biopsy specimens, not only the length but also the width of the biopsy core. Thin needles (20-22 gauge needles) are not adequate for fibrosis assessment since they often transect the portal tracts and may lead to false interpretation of the fibrosis stage [44]. Therefore, use of a 16-gauge needle is recommended and is usually considered as adequate. It should be noted that in case of cirrhosis or advanced fibrosis, counting the number of portal tracts is virtually impossible since they are all mixed in the fibrous septa. 
The TJLB has been proposed as a useful method to obtain "adequate" samples as it allows for more than one pass without any significant risk of bleeding. TJLB with three passes produces optimal biopsies for diagnostic purposes, which are adequate for staging and grading in $38 \%$ (biopsy length $\geq 25 \mathrm{~mm}$ ) or $25 \%$ ( $\geq 11$ portal tracts) of cases. [45, 46]. Another factor that needs consideration is the effect of processing on biopsy length. The core length is significantly shorter in the cartridge (23\%) compared to when removing it out on the tray, and it shrinks (7\%) again when subjected to formalin fixation and paraffin embedding [47]. Issues of smaller sample size and fragmentation problems have been overcome since aspiration techniques have given way to 'Tru-Cut' TJLB methods [48]. Although TJLB specimens yield decreased number of portal tracts, when contrasted with the risk of percutaneous biopsy in those with contraindications, TJLB provides a valuable alternative for assessment of liver histology. Consideration should be given to performing four passes if an assessment of disease severity is the goal.

There has been increasing interest in quantitative estimation of liver fibrosis, unlike the routine semiquantitative scores/staging systems used. Using techniques such as digital image analysis to assess the amount of collagen as a proportion of the total liver core, the collagen proportionate area (CPA) is calculated. The size of LB that is adequate for such quantitative estimations was recently assessed by Hall et al. [49]. They observed that increasing sample size increases the reliability of CPA results. Because an adequate LB only represents approximately $1 / 50,000$ th of the liver tissue, sampling variability can never be completely eliminated. In order to minimize its effect, large cohorts of patients and biopsies should be assessed in trials [50]. This will reduce the variation, which will then be random and multidirectional, and of a lesser consequence.

In order to minimize false differences in the scoring of paired biopsies, those of comparable size should be used in trials studying fibrosis progression or the effect of antiviral therapy. Clinical trials should also specify the minimum sample size considered adequate for inclusion. The histology report for LB gives a statement about the adequacy of the specimen in the form of the length and number of portal tracts included [51]. If the biopsy adequacy is questionable, the clinician should be made aware of this.

\section{Consensus statements}

- Biopsy length of $<10 \mathrm{~mm}$ and/or $<6$ portal tracts should be considered inadequate for fibrosis staging (B1).

- Biopsies of $\geq 20 \mathrm{~mm}$ or $\geq 11$ portal tracts should be considered ideal (B1).
- Final judgement on the adequacy of a sample for staging is based on the judgement of an experienced pathologist (C2).

- All histology reports should mention the sample size and the number of complete portal tracts included and comment on the adequacy of the sample, with the exception of cases with cirrhosis or advanced fibrosis (B1).

- Clinical trials should specify minimum sample size as an inclusion criterion (B1).

\section{Training for liver biopsy}

All LB techniques require specific training so as to ensure appropriate-sized specimen retrieval and the lowest rate of complications. The number of biopsies needing to be performed to become adequately trained is unknown. The AASLD's Training and Workforce Committee has recommended that the minimum number of procedures required to achieve training proficiency in standard transthoracic percutaneous LB is 40 (supervised) biopsies [12]. The number to become an expert will vary depending on the skill of the operator, the educator and specifics of the training setting. Training in US should also be developed as part of the required training for percutaneous LB to be able to do US-guided biopsy.

\section{Consensus statements}

- Specific training for LB is essential (A1).

- Although the required number of biopsies performed to become adequately trained is unknown, it is recommended that operators perform at least 40 biopsies (C2).

- Training in US should be a part of the required training for percutaneous LB (C2).

\section{Morphological evaluation of liver fibrosis: Current methods and scoring systems}

Descriptive reporting is often used when assessing diagnostic biopsies from an individual patient. However, in the trial and research setting, evaluation of biopsies is carried out using semiquantitative scoring systems that produce shorthand values for various categories of inflammation (grade) and fibrosis and architectural disruption (stage). The Ishak or "revised Knodell" system attempts to correct the criticism of numerical discontinuity by reintroducing the number 2 [52-54]. In other words, the Knodell system classified fibrosis into stages 0, "no fibrosis;" 1, "portal fibrosis;" 3, "bridging fibrosis;" 4 "cirrhosis" with the absence of stage 2, which describes "portal fibrosis with 
septae outside the portal area." This stage 2 was included in the revised system. The Ishak system assesses fibrosis in seven categories, ranging from normal to cirrhosis, and so has potentially more discriminant descriptive power. The French METAVIR Cooperative Study Group proposed a five-tier scoring system for hepatic fibrosis (from F0 to F4) and activity (from A0 to A4), which is less accurate than the Ishak score, but more reproducible between pathologists [55].

All scoring systems use the same principles to record liver disease stage. It is obvious that the "stage" or "fibrosis" score is composed of a mixture of features (fibrosis amount, location of fibrosis, architectural distorsion), none of which specifically depends on the amount of fibrous tissue in a LB sample. Computer-assisted image analysis of histochemically stained sections is a method for measuring fibrosis morphologically. It does not hinder other necessary diagnostic evaluations, and collagenous structures irrelevant to the disease process (which contribute to the variability between samples) can be excluded [56]. Considering any scoring system, the experience of the histopathologist is an important variable that must be considered and optimized [57].

\section{Future evolving techniques of morphological evaluation of liver fibrosis}

To reduce the potential inaccuracies in the processing and evaluation of biopsy specimens, several innovations have been made, which include incorporation of digital image analysis, genetic investigations and immunohistochemistry for functionally important molecules such as cytokines, cell cycle markers and viral life cycle markers into everyday practice.

\section{Virtual microscopy}

The virtual microscopy can be performed in two different ways. Interactive virtual microscopy by whole-slide imaging leaves the conclusion in the hands of pathologist. It significantly changes the working tools from optical microscopy and subjective decisions to computer screens and objective measurements. The automated virtual microscopy is even more exciting as computer systems evaluate the diagnoses. In liver pathology, the software has been developed for assessment of steatosis, fibrosis, necroinflammatory changes and hepatocyte volume.

Broadly speaking, image analysis of fibrosis assessment uses segmentation of digital images to measure the area of collagen and the area of tissue, producing a fibrosis ratio or CPA $[58,59]$. To quantify hepatic collagen histologically, it is necessary first to stain it specifically. Sirius red has an affinity for most hepatic collagens, including types I and III (the major components of liver collagen). Thus, Sirius red staining is the preferred histochemical method when quantifying liver fibrosis. However, some investigators have used reticulin or trichrome stains or immunohistochemistry, but due to variability in staining and difficulty in accurate thresholding, these techniques can suffer from poor reproducibility.

Image analysis studies have looked at fibrosis in many conditions, producing overall similar results ranging from 1 to $4 \%$ fibrous tissue in normal liver to $15-35 \%$ fibrous tissue in cirrhosis [60]. CPA correlates well with late stages of fibrosis, but is highly sensitive to sample size [43]. Problems with virtual microscopy include inter-observer differences and poor correlation of the fibrosis area with staging scores [61]. In addition, the digital data do not allow differentiation between low stages of fibrosis [62]. Clinical applicability of CPA is still being critically evaluated.

Another method called qFibrosis for LB assessment has been described, based on the strategy of combining pathology-relevant collagen architectural features with automated computer-aided tools. With input of imaging data from a liver sample, qFibrosis can automatically compute the fully quantitative fibrosis scores based on the respective collagen architectural features. qFibrosis can reliably stage liver fibrosis with reduced variability of sampling error and inter- and intra-observer bias in the assessment of both animal samples and CHB core biopsies. In addition, qFibrosis can differentiate late fibrosis stages on the Ishak scoring system, which suggests a potential to aid the monitoring of intra-stage cirrhosis changes. qFibrosis has demonstrated superior performance to CPA [63].

\section{Functional liver tissue analysis in LB}

The diagnostic evaluation of LB is mostly based on a panel of histochemical stains. These techniques may be complemented by various "- omic" tools to gain more data on the function of liver cells [64]. The cytokines, inflammatory mediators, viral proteins, cell cycle proteins and apoptosis markers and metabolic pathways can be investigated.

- Cytokine expression can be analyzed, e.g., TGF, EGFR, IL-6 and others. Due to the complex nature of cytokine action, a wide spectrum of different molecules and their receptors must be analyzed in detail to avoid insignificant or contradictory results. This needs virtual microscopy and digital image analysis.

- Proteomic studies including immunohistochemistry in LB have targeted cell structure-associated proteins (e.g., actin, tropomyosin, transgelin and human 
microfibril-associated protein 4) in order to identify biomarkers of liver cirrhosis [65].

- Cell cycle analysis can add valuable information, and viral antigens including hepatitis $\mathrm{C}$ antigen can be detected in liver tissue by immunohistochemistry $[66,67]$.

- DNA microarray technology has enabled genome-wide analysis of gene transcript levels. This technology has been applied in order to compare gene expression profiles at different stages of CHC [68], chronic hepatitis and autoimmune liver disease [69].

- Transcriptome analysis has shown prognostic value, e.g., in order to predict the severity of fibrosis progression after liver transplantation in patients with recurrent $\mathrm{HCV}$ infection [70]. Metabolic pathways and inflammatory cells can also be studied [71].

\section{Consensus statement}

- The combination of semiquantitative and morphometric assessment of fibrosis in LB should be considered for research purposes (B2).

\section{Blood tests for liver fibrosis assessment}

\section{Mechanisms of liver fibrosis and potential targets of fibrosis blood biomarker research}

Fibrogenesis is a complex process, usually triggered by an insult to the liver that leads to replacement of liver tissue by scar tissue. The process can take many years although acceleration of the process can occur if there is massive liver injury. Cell death can be caused by a variety of insults including viral, autoimmune, ischemic, toxic or metabolic. Each disease has different pathways to liver damage [72]. These processes trigger myofibroblasts, the effector cells of fibrogenesis, to secrete extracellular matrix. Excessive fibrogenesis is the result of an imbalance between the degradation and formation of extracellular matrix (ECM) components. These ECM macromolecules are mainly fibrous proteins with structural and adhesive functions, such as collagens and proteoglycans. During fibrogenesis, type I and III collagen levels increase up to eight times with a significantly higher increase of type I collagen than of type III collagen, changing the I/III ratio from 1:1 in the healthy liver to $2: 1$ in the cirrhotic liver. The liver also contains other collagen types, all of which may be involved in the excessive fibrogenesis during fibrosis, leading to the end result of cirrhosis with a total of up to six times more collagen than in the normal liver [73]. In the normal liver, the net amount of proteoglycans is low, whereas the level of several proteoglycans (decorin, biglycan, perlecan and syndecan) is increased during liver fibrosis [74]. An increase in collagen and proteoglycan can result from increased protein production, impaired protein degradation, diminished matrix degradation or a combination of these mechanisms. In the extracellular space, matrix degradation occurs predominantly as a consequence of the action of matrix metalloproteinases (MMPs). MMPs are secreted from cells into the extracellular space as proenzymes, which are then activated by a number of specific, usually cell surface-associated, cleavage mechanisms. The active enzymes are in turn inhibited by a family of tissue inhibitors of metalloproteinases (TIMP) [75]. By this combination of mechanisms, ECM degradation is closely regulated, which prevents inadvertent tissue damage. As a consequence, excessive matrix deposition may be the consequence of either increased formation or decreased degradation of connective tissue components or both. The stages of fibrogenesis can be arbitrarily divided into three stages-initiation, perpetuation and resolution. All these processes and variables are closely related. The ECM plays a critical role as in the cytokine milieu in which the central agent is $\mathrm{TGF}_{\beta}$. Consequently, this heterogeneity in the type of insult, stage of fibrogenesis, extent of fibrogenesis and type of immune response makes identifying biomarkers to stage fibrosis challenging.

\section{Classification of liver fibrosis blood biomarkers: an overview}

Gressner et al. separated biomarkers into class I and class II and published the most comprehensive classification of fibrosis biomarkers. [76]. Class I biomarkers are those intended to reflect ECM turnover and/or fibrogenic cell changes. Class II involves indirect serum biomarkers based on algorithmic evaluations of commonly observed functional alterations of the liver that do not necessarily reflect ECM turnover and/or fibrogenic cell changes. Class I biomarkers, in particular, originating from different lines of basic research and their changes, might be very difficult to interpret from a clinical or pathophysiological perspective.

The BIPED (burden of disease, investigative, prognostic, efficacy of intervention and diagnostic) classification categorizes biomarkers according to key parameters that are needed for assessment of clinical trials in research and development. Several classes have been defined:

1. Biomarkers that describe the progression of disease and correlate with known clinical parameters (burden of disease-B).

2. Biomarkers that capture the effect of an intervention in both known and unknown biological mechanisms associated with clinical outcome and that can act as a 
surrogate marker, i.e., changes in these biomarkers can predict the clinical outcome (efficacy-E).

3. Diagnostic biomarkers enabling identification of patients within the population and identification of subgroups within the diseased population (diagnostic-D).

4. Biomarkers that identify subjects with high risk of progression (prognostic-P)

5. Biomarkers not fully validated in a study population and therefore can be used solely for scientific investigations (investigatory-I).

Classification by the BIPED system may enable researchers from different backgrounds to communicate in a robust assessment framework, with a special focus on diagnostic, prognostic and possible burden of disease evaluations [77].

\section{Serum markers for diagnosis of liver fibrosis}

\section{Class I biomarkers}

Individual tests in general are too insensitive to be used, and hence class I biomarkers are often tested in panels. Only two test panels that underwent significant clinical evaluation. The first is MP3, which comprises amino-terminal propeptide of procollagen type III (PIIINP), a marker of fibrogenesis, and MMP-1. However, few studies have utilized this MP3 test and are limited to patients with chronic HCV infection [78]. The second is the enhanced liver fibrosis (ELF) test, which has been extensively tested in a variety of liver diseases. The ELF test comprises hyaluronic acid (HA), a component of the ECM, TIMP-1 and PIIINP reflecting collagen synthesis at the site of disease. A meta-analysis of its performance is summarized in Table 6 [79, 80]. Recently, real-time measurement of Wisteria floribunda agglutinin positive human Mac-2binding protein $[\mathrm{WFA}(+)-\mathrm{M} 2 \mathrm{BP}]$ and monitoring of timecourse changes in its level can identify patients at high risk of HCC development and can predict hepatic fibrosis [81, 82]. However, this is not available for wide use in many countries and warrants further studies in comparison to other non-invasive modalities.

\section{Class II biomarkers}

These can be divided into simple panels, which are derived from routine laboratory tests or patented panels, which are commercial kits of a large panel of tests. Meta-analyses of studies were limited, and a search found two studies $[83,84]$. Poynard et al. examined biomarkers of liver fibrosis, and his main objective was to examine the diagnostic utility of the biomarker for advanced fibrosis defined as $\geq$ F2 (METAVIR system) based entirely on the AUROC curve [83]. The study also adjusted for spectrum bias by using DANA analysis. There were 62 quality measures, and validation was based on the number of studies and number of patients. Among 2237 studies, 14 biomarkers were found to be validated, 9 were not patented, and 5 were patented (Tables 7 and 8). FibroTest (FT) was the most studied test with 33 different populations including 6549 patients and 925 controls. The mean diagnostic value for the diagnosis of advanced fibrosis ( $\geq$ F3) assessed using AUROC curves was 0.84 [95\% confidence interval (CI), 0.83-0.86], without significant difference between the causes of CLD, hepatitis $\mathrm{C}$, hepatitis $\mathrm{B}$, alcoholic or NAFLD. High-risk profiles of false-negative/-positive of FT were present in $3 \%$ of populations (mainly those with Gilbert syndrome, hemolysis and acute inflammation). FT had higher accuracy than the most used nonpatented test, APRI. No significant difference has been observed among the five patented tests [83].

Health authorities in some countries have already approved the use of validated biomarkers as the first-line procedure for the staging of liver fibrosis. This overview of evidence-based data suggests that biomarkers could be used as an alternative to LB for the assessment of fibrosis stage in the above four liver diseases, and neither biomarkers nor LBs are sufficient alone to make a definitive decision in a given patient, and all the clinical and biological data must be taken into account [83]. An examination of the Poynard et al. [83] meta-analysis finds that this is not a typical meta-analysis and the methodology is not clearly described. Data were not pooled for many of the tests, and when comparisons were made, pooling was

Table 6 Performance of the enhanced liver fibrosis (ELF) test in liver disease

\begin{tabular}{llll}
\hline Test/pooled estimates & Significant fibrosis $(\geq \mathrm{F} 2)$ & Severe fibrosis $(\geq \mathrm{F} 3)$ & Cirrhosis \\
\hline Sensitivity & $83 \%(95 \%$ CI $0.8-0.86)$ & $78 \%(95 \%$ CI 0.74-0.81) & $80 \%(95 \%$ CI 0.75-0.85) \\
Specificity & $73 \%(95 \%$ CI 0.69-0.77) & $76 \%(95 \%$ CI 0.73-0.78) & $71 \%(95 \%$ CI 0.68-0.74) \\
LR (+) & $4.00(95 \%$ CI 2.03-6.39) & 4.39 (95\% CI 2.76-6.97) & $3.13(95 \%$ CI 2.01-4.87 \\
LR (-) & $0.24(95 \%$ CI 0.17-0.34) & 0.27 (95\% CI 0.16-0.46) & 0.29 (95\% CI 0.19-0.44) \\
Diagnostic OR & $16.10(95 \%$ CI $8.27-31.34)$ & 16.01 (95\% CI 7.15-35.82) & 14.09 (95\% CI 5.43-36.59) \\
AUROC & 0.8813 & 0.8696 & 0.8770
\end{tabular}

$O R$ odds ratio, $L R$ likelihood ratio, $C I$ confidence interval, $A U R O C$ area under the receiver-operator characteristic curve 
Table 7 List of biomarkers from Poynard et al.'s meta-analysis [83]

\begin{tabular}{llll}
\hline Non-patented biomarkers & & Patented biomarkers & \\
PGA index & PI, GGT, ApoA1 & FT/FS (Fibrotest/fibrosure) & A2M, haptoglobin, ApoA1, bilirubin, GGT, age, gender \\
AP index (age-platelets) & Plt, age & FSP (Fibrospect II) & A2M, HA, TIMP-1 \\
Bonacini index & Plt, ALT, AST & ELF (enhanced liver fibrosis) & HA, PIIINP, TIMP-1 \\
Pohl score & Plt, AST & FM (Fibrometer) & Plt, AST, A2M, HA, PT, age, gender \\
Forns index & Plt, cholesterol, age & HS (Hepascore) & A2M, HA, GGT, age, gender \\
APRI & Plt, AST & & \\
MP3 index & PIINP, MMP1 & & \\
FIB-4 (Fibrosis-4) & Plt, AST, ALT, age & & \\
Fibroindex & Plt, AST, $\gamma$-globulins & & \\
\hline
\end{tabular}

ApoA1 apolipoprotein A1, $A 2 M$ alpha-2 macroglobulin, $A L T$ alanine transaminase, $A S T$ aspartate transaminase, $A P R I$ AST/platelet ratio index, GGT gamma glutamyle transferase, $H A$ hyaluronic acid, MMPs matrix metalloproteinase, PIIINP amino-terminal propeptide of procollagen type III, Plt platelets, $P T$ prothrombin time

Table 8 Summary statistics from Poynard et al.'s meta-analysis [83]

\begin{tabular}{llll}
\hline Test/pooled estimates & $\begin{array}{l}\text { Significant } \\
\text { fibrosis }(\geq \mathrm{F} 2)\end{array}$ & Differences between tests & Mean difference \\
\hline FT/FS (Fibrotest/fibrosure) & O.53-0.91 & FT $(0.83 ; 95 \%$ CI: 0.80-0.85) versus & $\begin{array}{c}0.06(95 \% \text { CI } 0.03-0.09 \text { without } \\
\text { heterogeneity; } p=0.0005)\end{array}$ \\
& & APRI $(0.76 ; 95 \%$ CI $0.73-0.80)$ & $0.02(95 \%$ CI $0.03-0.07 ; p=0.30)$ \\
FSP (Fibrospect II) & $0.83-0.88$ & & \\
ELF (enhanced liver fibrosis) & $0.77-0.94$ & FT and FM & $-0.005(95 \%$ CI $0.05-0.04 ; p=0.81)$ \\
FM (Fibrometer) & $0.78-0.90$ & FM and HS & $0.04(95 \%$ CI $0.01-0.08 ; p=0.13)$ \\
HS (Hepascore) & $0.76-0.85$ & & \\
\hline
\end{tabular}

APRI AST/platelet ratio index

used; hence, there were many inconsistencies in the description of the results.

Udell et al. [84] examined routine markers to assess their clinical utility to determine the presence of cirrhosis, regardless of underlying disease, a somewhat different question from that of the Poynard's study. The performance characteristics of clinical examination, individual laboratory tests and combination indices or models were summarized in the paper as sensitivity, specificity and LR (positive and negative) (Tables 9, 10). The overall prevalence of cirrhosis in the studies examined in the Udell meta-analysis was $24 \%$ (95\% CI 20-28\%), making spectrum bias less likely [84].

Overall, none of the meta-analyses were able to satisfactorily address the issue of the best performing biomarker. Consequently, indices derived from routine investigations provide only moderate accuracy for diagnosis or exclusion of cirrhosis and seem to be only slightly better than clinical evaluation. The performance of patented tests has been more thoroughly examined, and the two widely tested ones are the FT and ELF test. The Fibrotest (FT) pooled estimate was significantly better than the AST/platelet ratio index (APRI), but the ELF seemed to have higher AUROC curve values although the LR ratios seem to perform in the same range as the routine indices. If we use the cutoff of positive LR $\geq 10$ as a benchmark of an excellent diagnostic test for cirrhosis or advanced fibrosis, none of the tests achieved this although the closest was the Bonacini index $>7$, with LR (+) of 9.4, $95 \%$ CI 2.6-37, with rather wide CIs. For exclusion of cirrhosis, an LR (-) cutoff of $\leq 0.1$ would be considered an excellent test, and only the Lok index (AST: ALT ratio <0.2) achieved this0.09 (95\% CI 0.03-0.31), again rather wide CIs.

\section{Consensus statements}

- Circulating serum biomarkers of liver fibrosis generally can give moderate estimates in the diagnosis or exclusion of significant fibrosis and liver cirrhosis (A2).

- Serum biomarkers may be used either stepwise or in combination with other non-invasive tests such as imaging or elastography to improve the accuracy of liver fibrosis (C1).

- FT and APRI are recommended as the preferred noninvasive tests to assess for the presence of cirrhosis, and APRI is preferred in resource-limited settings (A1). 
Table 9 Formula for calculation of the indices from Udell et al. [84]

\begin{tabular}{|c|c|c|c|c|}
\hline Index & \multicolumn{4}{|c|}{ Formula } \\
\hline APRI & \multicolumn{4}{|c|}{$($ AST/upper limit of normal AST $) \times(100 /$ platelet count $[\times 103 / \mu 1])$} \\
\hline \multirow{8}{*}{$\begin{array}{l}\text { Bonacini cirrhosis discriminant score } \\
\text { (CDS) or Bonacini index }\end{array}$} & \multicolumn{4}{|c|}{ Platelet score + ALT: AST ratio score + INR score } \\
\hline & Score & Platelets $\left(\times 10^{3} / \mu \mathrm{L}\right)$ & ALT:AST ratio & INR \\
\hline & 0 & $>340$ & $>1.7$ & $<1.1$ \\
\hline & 1 & $280-340$ & $1.2-1.7$ & $1.1-1.4$ \\
\hline & 2 & $220-279$ & $0.6-1.19$ & $>1.4$ \\
\hline & 3 & $160-219$ & $<0.6$ & \\
\hline & 4 & $100-159$ & & \\
\hline & 5 & $40-99$ & & \\
\hline Lok index & \multicolumn{4}{|c|}{ Log odds $=-5.56-\left(0.0089 \times\left[\right.\right.$ Platelet count $\left.\left[\times 10^{3} / \mu 1\right]\right)+(1.26 \times$ AST: ALT ratio $)+(5.27 \times$ INR $)$} \\
\hline
\end{tabular}

$A L T$ alanine transaminase, $A S T$ aspartate transaminase, $A P R I$ aspartate transaminase platelet ration index, $L R$ Likelihood ratio, INR international normalized ratio

\section{Conventional radiological modalities for fibrosis assessment}

\section{Conventional ultrasound}

The use of conventional US has been established in diagnosing cirrhosis and differentiating chronic hepatitis from cirrhosis. Several studies have been performed to assess liver fibrosis stages using various US parameters, such as liver size, the bluntness of the liver edge, the coarseness of the liver parenchyma, liver surface nodularity, spleen size, etc. [85, 86].

\section{Normal versus cirrhotic livers on ultrasound}

The normal liver is homogeneous, containing fine-level echoes, and is either minimally hyperechoic or isoechoic compared to the normal renal cortex. Most cases of chronic hepatitis have a normal US examination. When cirrhosis develops, US may demonstrate a coarsened echotexture and other morphologic changes. Cirrhosis can be classified as micronodular $(0.1-1 \mathrm{~cm}$ in diameter) or macronodular (varying nodule size up to $5 \mathrm{~cm}$ in diameter). The sonographic patterns associated with cirrhosis include [87]:

\section{Volume distribution}

In the early stages of cirrhosis, the liver may be enlarged, whereas in the advanced states, the liver is often small, with relative enlargement of the caudate, left lobe or both, in comparison with the right lobe. Several studies have evaluated the ratio of the caudate lobe width to the right lobe width (C/RL) as an indicator of cirrhosis [88]. A C/RL value of 0.65 is considered indicative of cirrhosis. The specificity is $100 \%$ but the sensitivity is low (43-84\%), indicating that the $\mathrm{C} / \mathrm{RL}$ ratio is a useful measurement only if it is abnormal.

\section{Coarse echotexture}

Diffuse liver disease frequently shows increased echogenicity and coarse echotexture, which is a subjective finding. Liver attenuation is correlated with the presence of fat and not fibrosis [89].

\section{Nodular surface}

Irregularity of the liver surface during routine scanning has been appreciated as a sign of cirrhosis when the appearance is gross or when ascites is present. The nodularity corresponds to the presence of regenerating nodules and fibrosis.

\section{Regenerating nodules (RNS)}

RNs represent regenerating hepatocytes surrounded by afibrotic septa. RNs tend to be isoechoic or hypoechoic with a thin echogenic border, which corresponds to fibrofatty connective tissue [90].

\section{Dysplastic nodules}

Dysplastic nodules or adenomatous hyperplastic nodules are larger than RNs (diameter $\geq 10 \mathrm{~mm}$ ) and are considered premalignant. They contain well-differentiated hepatocytes, a portal venous blood supply and also atypical or frankly malignant cells.

\section{Other abnormalities}

Other abnormalities in cirrhosis include portal hypertension with its secondary signs of splenomegaly, ascites and portosystemic venous collaterals. 
Table 10 Summary findings from the Udell et al. study [84]

\begin{tabular}{lcc}
\hline Test/pooled estimates & LR $(+)$ & LR $(-)$ \\
\hline $\begin{array}{l}\text { Clinical impression of cirrhosis } \\
\text { Clinical impression of no cirrhosis }\end{array}$ & $4.8 ; 95 \%$ CI $2.5-7.2$ & $0.52 ; 95 \%$ CI $0.33-0.71$ \\
$\begin{array}{l}\text { Lok index (AST: ALT ratio }>1) \\
\text { Lok index (AST: ALT ratio }<0.2)\end{array}$ & $4.6 ; 95 \%$ CI 2.6-6.5 \\
$\begin{array}{l}\text { APRI }>2 \\
\text { APRI }<1\end{array}$ & $4.6,95 \%$ CI 3.2-6.0 & $0.09(0.03-0.31)$ \\
$\begin{array}{l}\text { Bonacini index }>7 \\
\text { Bonacini index }<3\end{array}$ & $9.4 ; 95 \%$ CI 2.6-37 & $0.33(0.23-0.43)$ \\
\hline $\begin{array}{l}A L T \text { alanine transaminase, } A S T \text { aspartate transaminase, APRI aspartate transaminase platelet ration index, } \\
L R \text { likelihood ratio, } C I \text { confidence interval }\end{array}$ &
\end{tabular}

\section{Liver fibrosis staging}

Although US can show a small, nodular liver in advanced cirrhosis, surface nodularity or increased echogenicity can also be seen in fatty liver without the presence of cirrhosis. US can identify diffuse parenchymal disease, but cannot reliably distinguish fat from fibrosis. Some investigators rely on a single US parameter to detect early cirrhosis (Table 11) [91], while the others try to test single or combined US parameters to distinguish each stage of significant fibrosis (F2-F4; Table 12) [92]. The use of conventional US to distinguish fibrosis stages remains problematic.

Liver fibrosis induces a progressive distortion of the intrahepatic vasculature and is accompanied by angiogenesis, concurring to portal hypertension in cirrhosis. Color Doppler US (CDUS) and power Doppler US (PDUS) are accurate in assessing the liver macrovascular anatomy and its changes secondary to portal hypertension, but no sign is observed before cirrhosis develops [93].

\section{Contrast-enhanced ultrasound}

Recently, contrast-enhanced ultrasound (CEUS) has become popular with the safety and reliability of a lessinvasive procedure. After the APASL 2009 consensus statement about hepatic fibrosis [1], several second-generation microbubble agents have become available, Definity (perflutren lipid microsphere; Lantheus Medical Imaging, Billerica, MA, USA), SonoVue (sulfur hexafluoride; Bracco, Milan, Italy) and Sonazoid (perfluorobutane; GE Healthcare, Oslo, Norway) [94]. Although there are some differences in the in vivo behavior and acoustic property of the microbubble, improvement of the imaging technique to demonstrate the dynamic and static microbubble has made it possible to increase the diagnostic ability for focal and diffuse liver disease [95]. There are two different ways to assess the microbubble behavior for evaluating hepatic fibrosis, the dynamic inflow/outflow microbubble and static accumulated microbubble. The transit time defined by the time intervals from vessel to vessel represents the parameter for evaluating the dynamic microbubble. The efficacy of transit time for grading hepatic fibrosis is controversial at present, and at least a definitive time point needs to be determined.

Overall, the evidence strongly suggests the clinical efficacy of CEUS for grading hepatic fibrosis. However, further studies are needed as the number of studies is relatively small; the definitive contrast parameter with the optimal kind of contrast agent has not yet been determined, and only few studies have compared the diagnostic performance between CEUS and other imaging modalities.

\section{Computed tomography}

One advantage of computed tomography (CT) imaging is that attenuation can be displayed in Hounsfield units. CT imaging has been primarily used in the diagnosis of focal liver lesions rather than diffuse liver diseases. Changes in attenuation on unenhanced CT imaging can be observed in some diffuse liver diseases. However, quantitative assessment of the density distribution of liver parenchyma showed that only diffuse steatosis and active alcoholic cirrhosis had significantly different mean hepatic attenuation values [96]. Contrast-enhanced CT using xenon gas or iodinated contrast agents can determine both global and local perfusion changes within the liver with high spatial and temporal resolutions. Normally hepatic parenchymal enhancement is minimal during the hepatic arterial phase (HAP), while during the portal venous phase (PVP) the normal hepatic parenchyma enhances markedly. The causes of enhancement during HAP are mainly localized hepatic lesions, but fibrosis or cirrhosis can also cause changes in hepatic attenuation due to associated hemodynamic changes [97]. Unfortunately, most contrast-enhanced CT studies involved patients with cirrhosis, and it is thus unclear whether changes in hepatic enhancement 
Table 11 Diagnostic performance of three ultrasound parameters to detect early liver cirrhosis, from Shen et al. [91]

\begin{tabular}{llllll}
\hline Parameters and cutoff value & Sensitivity & Specificity & Accuracy & PPV & NPV \\
\hline Length of spleen $(12.1 \mathrm{~cm})$ & 0.600 & 0.753 & 0.737 & 0.198 & 0.948 \\
Splenic vein diameter $(8 \mathrm{~mm})$ & 0.600 & 0.781 & 0.765 & 0.220 & 0.950 \\
Portal vein diameter $(12 \mathrm{~mm})$ & 0.767 & 0.446 & 0.475 & 0.124 & 0.949 \\
\hline
\end{tabular}

$P P V$ positive predictive value, $N P V$ negative predictive value

Table 12 Diagnostic performance of ultrasound parameters to detect significant fibrosis ( $>$ F2) from Choong et al. [92]

\begin{tabular}{llllllll}
\hline Ultrasound feature & Surface $(\mathrm{S})$ & Edge $(\mathrm{E})$ & Texture $(\mathrm{T})$ & $(\mathrm{S})+(\mathrm{T})$ & $(\mathrm{S})+(\mathrm{E})$ & $(\mathrm{T})+(\mathrm{E})$ & All scores \\
\hline Cutoff score & $\geq 1$ & $\geq 1$ & $\geq 1$ & $\geq 2$ & $\geq 1$ & $\geq 1$ \\
Sensitivity & $57(44-69)$ & $15(8-26)$ & $41(29-54)$ & $29(19-42)$ & $60(47-72)$ & $38(24-54)$ & $32(21-45)$ \\
Specificity & $62(51-72)$ & $90(83-85)$ & $72(62-81)$ & $88(79-94)$ & $57(46-67)$ & $83(75-89)$ & $85(76-91)$ \\
PPV $(\%)$ & $51(39-63)$ & $53(29-76)$ & $52(38-66)$ & $63(44-80)$ & $50(39-62)$ & $47(30-64)$ & $60(422-76)$ \\
NPV $(\%)$ & $67(55-77)$ & $60(51-68)$ & $64(53-73)$ & $64(54-72)$ & $67(55-77)$ & $77(68-84)$ & $64(54-72)$ \\
Accuracy & $59(51-67)$ & $53(45-61)$ & $57(49-65)$ & $59(51-67)$ & $60(51-67)$ & $61(53-69)$ & $59(51-67)$ \\
$p$ value & 0.03 & 0.32 & 0.07 & 0.04 & 0.03 & 0.02 & 0.05
\end{tabular}

Results are expressed as \% (95\% confidence interval)

$P P V$ positive predictive value, $N P V$ negative predictive value

could be used to diagnose mild or moderate hepatic fibrosis. CT can assess hepatic perfusion as well. Hepatic, aortic and portal venous time-density curves can be fitted. Using a dual-input one-compartment model to calculate the liver perfusion, arterial fraction, distribution volume and mean transit time, patients with cirrhosis could be differentiated from healthy controls with reasonable accuracy and perfusion parameters correlated with disease severity [98].

Contrast-enhanced methods cannot be performed on patients with renal failure or contrast allergy. Newer vascular specific contrast agents are being developed, which could lead to the development of simpler and better methods [99]. In a study evaluating the diagnostic accuracy of cirrhosis by imaging modalities (CT, MRI and US) compared to results obtained from histopathological diagnoses of resected specimens, it was found that irregular or nodular surface, blunt edge or morphological changes in the liver were the best predictive signs for cirrhosis on US, whereas liver parenchymal abnormalities, manifestations of portal hypertension and morphological changes in the liver were the best predictive signs on MRI and CT by multivariate analysis. MRI and CT were slightly superior to US in predicting cirrhosis, especially regarding sensitivity [100]. In a recent retrospective analysis, it was found that the $1 \mathrm{~d} / \mathrm{crl}$ ratio [sum of liver vein diameters (ld) divided by the caudate-right lobe ratio (crl-r)] $<24$ showed a sensitivity of $83 \%$ and a specificity of $76 \%$ for pre-cirrhotic liver fibrosis. Cirrhosis could be detected with a sensitivity of $88 \%$ and a specificity of $82 \%$ if the ld/crl ratio is $<20$ [101].

\section{Magnetic resonance imaging (MRI)}

MRI has become a mainstay of liver evaluation as it can be used in a comprehensive manner to (1) examine liver morphometry and function, (2) evaluate focal lesions and (3) by use of different contrast media provide additional information on perfusion and liver function. However, in the evaluation of liver fibrosis and cirrhosis, the ability of MRI to accurately assess this is not well established. Specific features on MRI including nodularity of the liver surface, hepatic vein narrowing, increased caudate to right lobe ratio and expanded gallbladder fossa reliably identify cirrhosis but mostly remain absent in earlier stages of fibrosis [102]. MRI techniques that have been utilized in the evaluation of liver fibrosis include the following:

\section{Contrast-enhanced magnetic resonance imaging}

Contrast agents are used to enhance the features of MRI. They can be divided into extracellular agents (e.g., gadolinium chelates) or reticuloendothelial agents [superparamagnetic iron oxides (SPIOs)]. They are synergistic in the evaluation of liver fibrosis. SPIOs darken spared liver, while gadolinium chelates tend to accumulate in the extracellular space associated with fibrosis and cause fibrosis to gain signal and appear as bright reticulations. Both agents can be used simultaneously, known as double contrast scanning.

With gadolinium chelates, a delayed, heterogeneous enhancement pattern is associated with bright-appearing reticulations suggestive of hepatic fibrosis. SPIO contrast 
agents lead to a reduced signal-to-noise ratio (SNR) in cirrhosis. SPIO contrast agents create hypointensity within the liver parenchyma based on its accumulation in reticuloendothelial cells and lead to accuracy of $85 \%$ in detecting liver fibrosis compared with histology. The sequential administration of gadolinium followed by SPIO contrast media yielded even greater accuracy (93\%) overall based on double-enhanced image scores to distinguish between mild and advanced fibrosis [103].

There is considerable variation in MRI of liver techniques, with few high-quality studies to be certain of their utility in the diagnosis of liver fibrosis. In addition, the use of these new contrast agents is expensive and time for scanning prolonged with breath-hold requirements that may not be suitable for elderly and unfit patients, making this not an optimal choice for evaluation of liver fibrosis.

\section{Diffusion-weighted magnetic resonance imaging}

The principle of diffusion-weighted magnetic resonance imaging (DWMRI) is that the observed signal intensity of tissue varies inversely with the freedom of water proton diffusion. With DWMRI, tissues with reduced water proton diffusion will be brighter than those with normal water proton diffusion. The sensitivity of the sequence for diffusion can be varied using various parameters, and this is known as the diffusion weighting to the "b" value. The apparent diffusion coefficient (ADC) of water protons in tissues is determined by the slope of the log intensity versus the $b$ value. As fibrosis increases, one expects that the ADC will decrease as the diffusion is reduced. Consequently, the ADC can be used as a quantitative measure of the extent of fibrosis. A number of studies have examined ADC as a measure of liver fibrosis compared to liver histology, but the best study is a high-quality meta-analysis evaluated with quality assessment for studies of diagnostic accuracy (QUADAS) comparing DWMRI and magnetic resonance elastography (MRE) to liver histology [104]. The pooled estimates inform us that DWMRI has a moderate accuracy, with relatively low sensitivity (below 0.8 ) for detection of $\geq \mathrm{F} 2$ fibrosis and $\geq \mathrm{F} 3$ fibrosis. DWMRI is limited in the assessment of liver fibrosis in the presence of fat and iron because of a reduction in the signal of the liver. Also the presence of fatty change is thought to affect diffusion; therefore, DWMRI may be limited in the assessment of fibrosis in NASH.

\section{Magnetic resonance spectroscopy}

Currently magnetic resonance spectroscopy (MRS) can be performed by analyzing signals from either hydrogen $\left({ }^{1} \mathrm{H}-\right.$ MRS) or phosphorus ( $\left.{ }^{31} \mathrm{P}-\mathrm{MRS}\right)$ atoms. ${ }^{1} \mathrm{H}$-MRS provides insights into metabolism, as phosphorus is associated with molecules involved in various metabolic processes. Although not specific for liver, anatomically the concentration of highly active metabolic cells makes it ideal for

${ }^{31} \mathrm{P}-\mathrm{MRS}$. This technology does not measure fibrosis but rather metabolism. Consequently, alterations in cell metabolism are an indirect measurement of liver fibrosis. In vivo MRS examining liver fibrosis has used 31P-MRS. After a standard MR imaging for localization, special MR pulse sequences are applied to generate spectroscopic data within the appropriate anatomical location and volume; a typical examination takes 45-60 min. To date, only small studies with heterogeneous populations have been studied [102-105]. Consequently, the role of MRS in diagnosis of liver fibrosis/cirrhosis remains undefined.

\section{Consensus statements}

- Conventional ultrasound cannot be used for the diagnosis of hepatic fibrosis (B2).

- Conventional ultrasound for diagnosis of early cirrhosis should be confirmed by additional studies (C1).

- Contrast-enhanced ultrasound with a second-generation microbubble agent is useful for staging hepatic fibrosis (C2).

- Conventional CT and MRI have higher specificity and sensitivity than conventional ultrasound for the diagnosis of cirrhosis (A1).

\section{Liver elastography techniques}

Liver elastography is one of the non-invasive methods for the evaluation of the severe hepatic fibrosis. Elastographic methods of the liver can be done using US waves or MRI.

\section{Ultrasound-based elastography}

Elastographic methods using US waves for the evaluation of liver fibrosis can be divided into:

1. Strain elastography (or quasi-static elastography):

2. Shear waves elastography (SWE):

(a) Transient elastography (TE; FibroScan)

(b) Point shear wave elastography (SWE) using acoustic radiation force impulse (ARFI) quantification including virtual touch quantification (VTQ) from Siemens and ElastPQ from Philips.

(c) 2D real-time shear wave elastography-supersonic shear imaging (SSI), the Aixplorer system and SSI, but also more recently the systems from General Electric and Toshiba. 


\section{Shear wave elastography (SWE)}

SWE calculates the tissue deformation or strain induced by a stress. The stress may be applied with a slight free hand compression, using the transducer, or may derive from endogenous movements, such as those generated by the heart or very large arteries during each cardiac cycle. The extent of the deformability of the tissue (strain) is related to its stiffness. The elastic ratio is the ratio of strain distribution in two selected regions of interest (ROIs). SWE is divided into the following three methods:

\section{Transient elastography (TE; FibroScan ${ }^{\circledR}$ )}

TE or FibroScan ${ }^{\circledR}$ measures liver stiffness using a section of liver that is approximately $1 \mathrm{~cm}$ in diameter and $5 \mathrm{~cm}$ long, which is 100 times greater in size than a standard LB, and thus may be more representative of the entire hepatic parenchyma [106].

Multiple studies have described test characteristics of US-based TE. At least three meta-analyses have been published [107-109]. The most recent (which included 50 studies) estimated test characteristics by reporting the AUROC curve [106]. The mean AUROC curves for the diagnosis of significant fibrosis, severe fibrosis and cirrhosis were $0.84,0.89$ and 0.94 , respectively (with a value of 1 corresponding to a perfect test). Estimates for the diagnosis of significant fibrosis were influenced by the type of underlying liver disease and the cutoff level for diagnosing cirrhosis. The appropriate cutoff levels may vary by the cause of the liver disease. The most consistent findings were in patients with CHC. An earlier meta-analysis of nine studies provided summary estimates in terms of sensitivity and specificity [107]. For diagnosing cirrhosis, the sensitivity was $87 \%$ and the specificity was $91 \%$. For diagnosing significant (F2-4) fibrosis, the sensitivity was $70 \%$ and the specificity was $84 \%$.

\section{Factors influencing LSM using FibroScan}

The factors that may reduce the accuracy of US-based TE include ascites, elevated central venous pressure (as in heart failure) and obesity because fluid and adipose tissue attenuate the elastic wave $[110,111]$. A probe has been developed (FibroScan XL probe) in an attempt to improve accuracy in obese patients. Studies suggest that it is able to accurately assess the degree of fibrosis, but that it may not be superior to the standard $M$ probe in obese patients [112-114]. Liver inflammation may also reduce the accuracy of the test [115]. Other factors that may influence the test results are sex (men have higher liver stiffness values than women), metabolic syndrome and operator experience [116]. It is not clear whether hepatic steatosis has an effect on accuracy [117-119].
Liver stiffness values may be associated with prognosis. In a meta-analysis of 17 studies with 7058 patients with chronic liver disease, baseline liver stiffness was associated with the risk of hepatic decompensation [relative risk (RR) $1.07,95 \%$ CI 1.03-1.11], HCC development (RR 1.11, $95 \%$ CI 1.05-1.18) and death (RR 1.22, $95 \%$ CI 1.05-1.43) [120]. A study of patients undergoing hepatectomy for HCC [121] showed that liver stiffness $>15.7 \mathrm{kPa}$ was a risk factor for postoperative liver failure (sensitivity $96 \%$ and specificity $69 \%$ ). Also, the use of FibroScan compared to LB has been evaluated in a meta-analysis with 8 published studies including 801 patients revealing higher liver stiffness cutoff with the use of FibroScan [122].

Liver stiffness measurement assessed by FibroScan is a reliable marker of severe liver fibrosis in primary sclerosing cholangitis (PSC). Stiffness increases significantly and exponentially over time. Liver stiffness and its progression rate are two major prognostic factors in PSC [123].

The limitations regarding FibroScan are related to its cost, its infeasibility in patients with ascites, its affection by elevated aminotransferase levels, the need for an increased number of unreliable measurements in patients with high BMI and its inability to distinguish patients without fibrosis or with mild fibrosis from those with mild or moderate fibrosis, respectively.

\section{Acoustic radiation force impulse imaging (ARFI)}

ARFI is a form of tissue elastography that is integrated into a conventional high-end ultrasound machine (Siemens S2000, Siemens, Erlangen, Germany) and ElastPQ from Philips. ARFI has the advantage of being a feature existing on an ultrasonography machine. This allows for the convenience of assessing for structural abnormalities, steatosis as well as fibrosis, in a single sitting. Two meta-analyses by the same group evaluated the performance of ARFI in staging liver fibrosis compared to LB and revealed good diagnostic accuracy of the ARFI imaging for the staging of $F \geq 2 \quad$ (AUROC $=87 \%$ ) and $F \geq 3 \quad$ (AUROC curve $=91 \%)$ and excellent diagnostic accuracy for $F=4 \quad$ (AUROC curve $=93 \%$ ) $[124,125]$. Another prospective study on 70 patients with different etiologies published in 2013 by Yap and colleagues showed that ARFI has strong correlation with higher fibrosis scores compared to lower, and when compared to a the above pooled meta-analysis cutoff values, the sensitivity and specificity for detecting true $F \geq 3$ are higher than those of $F \geq 2$ [126]. ARFI elastography had a very good positive predictive value $(93.2 \%)$ for predicting the presence of significant fibrosis and excellent negative predictive value $(97.8 \%)$ for excluding the presence of compensated liver cirrhosis [127], although the accuracy was less in staging hepatic fibrosis in patients with NAFLD as shown by 
another meta-analysis [128]. When compared to TE, ARFI has been shown in another two meta-analyses to have a similar predictive value for significant fibrosis and cirrhosis, and both had better accuracy than real-time elastography (RTE) in predicting cirrhosis [129, 130].

\section{Supersonic shear imaging (SSI)}

SSI is a simple, accurate, reliable, non-invasive method of assessing liver fibrosis [131]. The mean \pm SD LS as measured by SSI in patients without known liver pathology was found to be $6 \pm 1.4$, slightly higher in men, and is not affected by BMI [132]. Many studies have shown that SSI and TE using an XL probe accurately quantified liver stiffness and fibrosis stage in obese chronic liver disease patients, including those with chronic HCV infection, compared with histologic analyses and ARFI [133-137]. However, SSI was shown to be a poor predictor of disease activity and steatosis level [136].

A recent study has assessed the clinical use of LSM evaluated by SSI, TE and ARFI in a cohort of NAFLD patients who underwent LB. The AUROCs for SSI, FibroScan and ARFI were 0.86, 0.82 and 0.77 for diagnoses of $\geq F 2 ; 0.89,0.86$ and 0.84 for $\geq F 3$; and $0.88,0.87$ and 0.84 for F4, respectively. SSI had a higher accuracy than ARFI for diagnoses of significant fibrosis $(\geq \mathrm{F} 2)$ $(p=0.004)$ [138].

Accuracy for diagnosing $F \geq 3$ and F4 was 89.6-94.7\% and 87.7-96.0 \%, respectively, in NAFLD subjects. The negative predictive values for diagnosing $F \geq 3$ and F4 were 94.2-96.6 and $100 \%$, respectively. Only portal fibrosis significantly correlated with an increased hepatic elastic ratio measured by real-time tissue elastography. The AUROC curve of the elastic ratio correlated better than serum fibrosis markers in both early and advanced fibrosis stages [116]. In patients with CHC, SWE has been found to be of better [133] or similar accuracy as TE [139]. In another study, SSI with ARFI had higher accuracy than FibroScan for the diagnosis of severe fibrosis ( $\geq F 3$; $p=0.0016)$ and higher accuracy than ARFI for the diagnosis of significant fibrosis $(\geq \mathrm{F} 2 ; p=0.0003)$, but no significant difference was observed for the diagnosis of mild fibrosis and cirrhosis [140].

\section{Consensus statements}

- TE is an established technique and is recommended as the initial assessment for significant liver fibrosis and cirrhosis (A1).

- TE helps to predict complications of cirrhosis and its prognosis (A2).

- The interpretation of results should be carefully weighted in the presence of limiting factors (A1).

- TE is a highly reproducible and user-friendly technique for assessing liver fibrosis in patients with CLD. However, because TE reproducibility is significantly reduced $(p<0.05)$ in patients with steatosis, increased BMI, lower degrees of hepatic fibrosis and narrow intercostal spaces, caution could be warranted in the clinical use of TE as surrogate for LB (A1).

- SSI is an accurate measure in predicting fibrosis stage and is as accurate as TE and ARFI in this respect (B2).

\section{Magnetic resonance elastography (MRE)}

MRE provides one of the more robust methods to measure liver stiffness. However, although it requires special software and equipment, it can be easily implemented on existing MR scanners with suitable alterations [141, 142]. MRE has been compared to TE [143] as well as other noninvasive markers of fibrosis [144]. To evaluate the diagnostic capabilities of MRE, two high-quality meta-analyses have been performed comparing MRE with histology as the gold standard and had clearly defined inclusion and exclusion criteria, both using QUADAS scoring to evaluate quality [104, 145] (Table 13).

Wang et al. [104] examined differences based on the following groupings using a $2 \times 2$ table: F0 versus F1-F4 (score G1), F0 and F1 versus F2-F4 (score G2), F0-F2 versus F3 and F4 (score G3) and F0-F3 versus F4 (score G4). All the point estimates were over $90 \%$ for the key comparisons, and the AUROC was 0.98 and 0.99 for group

Table 13 Pooled estimates of diagnostic parameters for magnetic resonance elastography from Guo et al. [145]

\begin{tabular}{lllll}
\hline Test/pooled estimates & F1 & F2 & F3 & F4 \\
\hline Sensitivity & $0.87(0.84-0.90)$ & $0.87(0.84-0.90)$ & $0.87(0.83-0.90)$ & $0.93(0.88-0.96)$ \\
Specificity & $0.93(0.88-0.97)$ & $0.94(0.91-0.97$ & $0.92(0.90-0.94)$ & $0.91(0.88-0.93)$ \\
LR (+) & $7.97(4.68-13.57)$ & $10.83(6.67-17.58)$ & $10.22(6.48-16.11)$ & $8.85(6.00-13.05)$ \\
LR (-) & $0.16(0.11-0.23)$ & $0.14(0.07-0.27)$ & $0.15(0.11-0.20)$ & $0.06(0.02-0.18)$ \\
Diagnostic OR & $56.1(28.9-108.9)$ & $116.2(50.4-267.7)$ & $97.7(50.8-187.7)$ & $184.8(47.4-720.4)$ \\
AUROC & 0.94 & 0.97 & 0.96 & 0.97 \\
\hline
\end{tabular}

$O R$ odds ratio, $L R$ likelihood ratio, $C I$ confidence interval, $A U R O C$ area under the receiver-operator characteristic curve 
Table 14 Pooled estimates of diagnostic parameters for magnetic resonance elastography (MRE) from Wang et al. [104]

\begin{tabular}{lll}
\hline Test/pooled estimates & F0 and F1 versus F2-F4 & F0-F3 versus F4 \\
\hline Sensitivity & $0.94(0.808-0.983)$ & $0.985(0.795-0.999)$ \\
Specificity & $0.953(0.872-0.984)$ & $0.939(0.869-0.972)$ \\
LR (+) & $20(7-57)$ & $16(7-36)$ \\
LR (-) & $0.06(0.02-0.22)$ & $0.02(0.00-0.26)$ \\
Diagnostic OR & $317(55-1796)$ & $1006(36-26,068)$ \\
AUROC & $0.98(0.97-0.99)$ & $0.99(0.97-0.99)$ \\
\hline
\end{tabular}

F1-F4 according to the METAVIR score

$O R$ odds ratio, $L R$ likelihood ratio, $C I$ confidence interval, AUROC area under the receiver-operator characteristic curve
2 and 4, making this test close to ideal. However, MRE studies did have high statistical heterogeneity and publication bias ([104]; Table 14). Guo et al. [145] showed that MRE was found to be more accurate than ARFI with a higher combination of sensitivity, specificity, LR and AUROC particularly in diagnosing early stages (F1-2) of hepatic fibrosis.

MRE as a technique has some significant advantages [141, 142]. It is generally well tolerated by patients except those with claustrophobia. It is not affected by fatty change in the liver, although inflammation (NASH) can affect the readings. It is not affected by ascites or obesity or bowel interposition between the liver and anterior abdominal wall. Furthermore, it can evaluate the entire liver rather than a focal region as in LB or TE. The main limitations of MRE [141] are the cost of the procedure, the requirement for high iron content in the liver as in the cases of hemochromatosis or hemosiderosis, and the requirement for consistent breathholding by the patient. MRE incorporated into routine MRI could provide a comprehensive evaluation of the liver, including fatty change, presence of focal lesions and PHT.

As MRE is proving to be an accurate and reliable test, many single etiology studies with MRE in diseases such as hepatitis B, hepatitis $\mathrm{C}$, alcoholic liver disease and NASH have emerged [141, 146-149], and all show high performance (accuracy $>0.90$ ) of MRE in the detection of fibrosis. Large population studies are still lacking. There were few studies comparing MRE to TE, but MRE had a higher technical success rate than TE and a better diagnostic accuracy (0.994 for $F>2$; 0.985 for $F>3 ; 0.998$ for $F>4)$ [143].

\section{Consensus statements}

- MRE is an emerging modality for accurate assessment for hepatic fibrosis and is recommended (A1).

- MRE is at least as accurate as ARFI in predicting hepatic fibrosis in patients with different etiologies (B2).

\section{Post-liver transplantation fibrosis}

Risk factors of post-transplant fibrosis include metabolic syndrome, post-transplant HCV recurrence with high viral load, advanced donor age, donor steatosis and prolonged warm ischemia time. What is unique for post-transplant patients is that the metabolic syndrome and its components are prevalent after liver transplantation compared to the non-transplant population. In this respect, factors related to the graft (donor's steatosis and warm ischemia time) and factors related to the type of immunosuppressive drugs (steroids and CNI) have been studied. Evidence supports the role of graft steatosis and older donor's age as important for post-transplant fibrosis progression regardless of the etiology of transplantation [150, 151], while no clear large-scale studies supported the role of one immunosuppressive over another.

\section{Assessment of fibrosis progression after liver transplantation}

Assessment of graft fibrosis and its progression are important for graft and patient survival. The rate of progression will define the need for modifying the type and dose of immunosuppressive drugs and also the need for aggressive treatment of factors related to the metabolic syndrome and/or starting antiviral treatment. Because many factors (such as rejection and infection) can affect the post-transplant laboratory tests, evidence did not support the use of such tests in assessing fibrosis progression in post-transplant situations. The validity of TE post-transplant is not yet clearly established. This is because the accuracy of TE readings is significantly reduced in the context of high transaminase levels, which are common in post-transplant patients because of associated pathologies such as acute cellular rejection (ACR), biliary obstruction or infection. Furthermore, graft steatosis and obesity are both common post-liver transplantation, and these factors also affect the accuracy of TE readings [111, 152]. Therefore, further validation of TE in diagnosis of post- 
transplant fibrosis is needed. LB remains the gold standard in assessment of post-transplant fibrosis and its progression. The issue is not only to biopsy or not, but also the time of biopsy and the findings needed to define the rate of fibrosis progression. Scheduled (protocol) LBs tailored according to the presence or absence of one or more of the risk factors are the most accepted modality by many studies $[11,153]$.

How best to determine rapid fibrosis progression (RFP) remains a key area of debate. Progression changes in the fibrosis stage on consecutive annual biopsies represent clinically significant fibrosis progression and indicate the need for intervention [153].

\section{Consensus statements}

- LB is the 'gold standard' for diagnosis and follow-up of post-transplant fibrosis. Protocol LB is of utmost importance in the post-transplant care of these patients (A1).

- Significant fibrosis progression is defined as the occurrence of progression on consecutive annual LBs or the presence of portal hypertension (A1).

- Significant fibrosis or portal hypertension 1 year after transplantation predicts rapid progression and graft loss and indicates urgent intervention (B1).

- Transient elastography for replacing biopsy in the assessment and diagnosis of post-liver transplantation fibrosis progression still needs validation through largescale clinical studies (C2).

\section{Cost-effectiveness of non-invasive markers for liver fibrosis}

\section{Cost-effectiveness studies comparing methods used for detecting and grading liver fibrosis}

As reported from many studies, non-invasive tests used in the diagnosis of liver fibrosis are still expensive in many countries. A decision analytic model was developed in a hypothetical patient population with $\mathrm{CHC}$ genotype 1 to assess the clinical and economic outcomes of non-invasive testing strategies in the diagnosis of significant liver fibrosis compared to LB [154]. The authors of this study included a testing algorithm using the FibroSure test, a non-invasive measure of fibrosis, followed by LB for patients with indeterminate results, FibroSpect II and FibroScan. From this study, in comparison with LB alone, there was a cost savings of $\sim \$ 770 /$ person with the Fibrosure testing algorithm, but a net decrease in accuracy of $14 \%$. FibroSpect II and Fibroscan had poorer accuracy (decreases of 12 and $4 \%$, respectively) and lower costs ( $-\$ 138$ and $-\$ 357$, respectively) compared to the FibroSure algorithm. In Canada, it was reported that the average costs for LB, FibroTest (FT) and FibroScan were \$1255, $\$ 350$ and $\$ 131$ (US\$), respectively [155]. The authors of this study suggested that, compared to LB, non-invasive testing algorithms can result in short-term cost savings, but the consequences of misdiagnosis in terms of health outcomes and treatment costs may outweigh the short-term gains in cost and convenience.

The total costs associated with the FT were compared to LB in patients with CHC [156]. The authors found that the FT alone strategy for men and women was US $\$ 193,979$ and US $\$ 213,525$, respectively. The total costs associated with the LB alone strategy for men and women were US $\$ 195,169$ and US $\$ 214,760$, respectively. The authors of this study concluded that in clinical settings where testing is required prior to treatment, FT alone is more effective and less costly than LB although the actual cost savings appear minimal and may have less value because of the decreased accuracy of FT [146]. The cost-effectiveness of TE performed annually was found to be better than LB performed every 7 years in the evaluation of liver fibrosis in patients with HIV/HCV coinfection [157].

Stevenson et al. [158] have conducted a systematic review and an economic evaluation of the diagnostic accuracy, cost-effectiveness and effect on patient outcomes of four non-invasive tests for liver fibrosis in patients with suspected alcohol-related liver disease. These tests include the ELF, FibroTest, FibroMAX and TE. The authors concluded that no conclusive result could be provided on the most cost-effective strategy until further data are available [158]. Canavan et al. [159] used a Markov decision analytic model to simulate a hypothetical cohort of 10,000 patients with chronic HCV initially without fibrosis over their lifetime and reported the cost-effectiveness of annual surveillance for cirrhosis in patients with chronic HCV. The authors reported that an annual definitive FibroScan is the optimal strategy to diagnose cirrhosis. The study also showed that annual FibroScan surveillance of 132 patients' results in the diagnosis of one additional HCC case over a lifetime. The incremental cost-effectiveness ratio for an annual definitive FibroScan is $£ 6,557.06 /$ quality-adjusted life years gained. The authors of this study concluded that an annual definitive FibroScan may be a cost-effective surveillance strategy to identify cirrhosis in patients with chronic $\mathrm{HCV}$, thereby allowing access of these patients to HCC screening.

From the above-mentioned studies and others, we can suggest that non-invasive tests seem to be more cost-effective in the assessment of liver fibrosis in patients with CHC compared to LB. However, there are several limitations of these studies that should be considered. To avoid 
the reported limitations of the studies, more research on the cost-effectiveness of non-invasive tests compared to LB in different areas and settings is needed. In addition, the costs in most studies are derived from US or European countries, and the cost-effectiveness may vary largely according to the cost from individual countries. This needs to be remembered when interpreting the cost-effectiveness of invasive and non-invasive tests in other parts of the world. However, according to the WHO guidelines, APRI is the most cost-effective non-invasive test to assess hepatic fibrosis compared to FT and TE.

\section{Consensus statements}

- There are many limitations to the economic studies that assessed the cost-effectiveness of non-invasive tests in evaluation of liver fibrosis (A1).

- So far, non-invasive tests are more cost-effective in the assessment of liver fibrosis compared to LB (B2).

- Compared to LB, FibroTest and TE are more costeffective (A2).

- In low economic countries, APRI is the most costeffective non-invasive test compared to FT and TE (A2).

- Studies on cost-effectiveness of non-invasive tests versus LB in different areas and settings are needed for policy decision makers (A1).

\section{Hepatic venous pressure gradient (hvpg) measurement for assessment of liver fibrosis}

\section{HVPG methodology and technique}

Hepatic vein catheterization, with measurements of the HVPG, is the preferred technique to estimate portal pressure and is rapidly becoming a routine test in many hospitals worldwide. It is an indirect method of assessing the portal pressure gradient in cirrhosis with a predominantly sinusoidal and/or post-sinusoidal site of resistance. It is well documented that HVPG measurement correlates well with direct portal pressure measurement, and the agreement is sufficiently good to use it as a surrogate measurement. The normal value of the HVPG is between 1 and $4 \mathrm{mmHg}$. HVPG reflects the severity of PHT, with $\geq 6 \mathrm{mmHg}$ defining PHT. Clinically significant PHT is defined by HVPG at and $>10 \mathrm{mmHg}$, as the development of ascites, varices and variceal bleeding usually occurs above this threshold [160].

Several studies have compared WHVP with portal venous pressure (PVP) in patients with cirrhosis but with relatively small cohorts and using different techniques for assessing PVP. Some of these studies found a moderate degree of correlation between the two measurements, especially in patients with alcoholic cirrhosis [161-163] and HCV cirrhosis [164]. Other studies reported the lack of significant correlation between the two techniques, especially in patients with non-alcoholic cirrhosis [165, 166]. Reproducibility in measuring WHVP per se has been questioned by Keiding et al. [167]. In this study, $61 \%$ of the HVPG measurements in two separate hepatic veins differed by as much as $4-34 \mathrm{mmHg}$. Assessment of the agreement between WHVP and PVP in cirrhotic patients done by Thalheimer et al. revealed that WHVP measurement correlates well with direct portal pressure measurement, and the agreement is sufficiently good to use this as a surrogate measurement [168]. Assessment by Thalheimer et al. showed that the range of difference between WHVP and PVP was from -8 to $9 \mathrm{mmHg}$ (mean $-0.4 \pm 2.67 \mathrm{mmHg}$, median $0 \mathrm{mmHg}$ ), and the coefficient of determination $\left(r^{2}\right)$ was 0.93 [166]. The agreement of WHVP with PVP in patients with non-alcoholic liver disease is also well documented in several studies. Assessment by Thalheimer et al. showed that the range of difference between WHVP and PVP was from -10.5 to $10 \mathrm{mmHg}$ (mean $-0.39 \pm 3.29 \mathrm{~mm} \mathrm{Hg}$, median $0 \mathrm{mmHg}$ ), and the coefficient of determination $\left(r^{2}\right)$ was 0.83 [169].

\section{Complications of HVPG measurement}

Only minor complications such as transient cardiac arrhythmias, local pain or vagal reaction have been reported, and these occur infrequently ( $<1 \%$ of patients). HVPG measurements can be performed in 10 min with TJLB through the same route. Despite its advantages such as safety, feasibility and reproducibility, the technique is invasive. The HVPG procedure shows low acceptance rates among patients with chronic liver disease and requires the technical expertise typically found at tertiary care centers [170].

\section{HVPG and liver fibrosis}

HVPG measurement can be used in the diagnosis of liver fibrosis, risk stratification, identification of patients with $\mathrm{HCC}$ who are candidates for liver resection, monitoring of the efficacy of treatment and assessment of progression of PHT. It has been proposed that serial HVPG measurements could assess fibrosis or cirrhosis irrespective of etiology. In the diagnosis of stage 1 compensated cirrhosis, the sensitivity and specificity of HVPG for predicting stage 1 compensated cirrhosis were 78 and $81 \%$ in $6 \mathrm{mmHg}$ of HVPG, respectively [171].

A positive correlation between the HVPG and fibrosis score has also been reported among chronically HBV 
infected patients [172]. HVPG predicted clinical decompensation in patients with compensated cirrhosis. Patients with HVPG $<10 \mathrm{mmHg}$ have a $90 \%$ probability of not developing clinical decompensation in a median follow-up of 4 years [160]. HVPG (AUROC 0.85; sensitivity: $80 \%$; specificity: $77 \%$ ) was superior to serologic biomarkers in predicting advanced fibrosis in chronic viral hepatitis [173]. In post-liver transplant patients, liver stiffness measurement (LSM) correlated with HVPG measurements, and the correlation between LSM and HVPG was excellent in patients with HVPG values $<10-12 \mathrm{mmHg}$ [174]. The AUROC curve for predicting HVPG $10-12 \mathrm{mmHg}$ ranges from 0.76 to 0.99 with a cutoff of $13.6-34.9 \mathrm{kPa}$ $[174,175]$. The correlation between portal pressure and LSM decreases when HVPG values exceed 10-12 $\mathrm{mmHg}$. HVPG $>6 \mathrm{mmHg}$ and HVPG $>10 \mathrm{mmHg}$ were predicted by 8.7 and $21 \mathrm{kPa}$ cutoffs, respectively, among post-LTx patients with HCV recurrence [176].

\section{Consensus statements}

- HVPG should be recommended for the screening of all cirrhotic patients and assessing the response to therapy (A2).

- HVPG correlates with the histological stage of fibrosis, but not beyond HVPG above 10-12 mmHg (A1).

- HVPG correlates with liver stiffness (B2).

- HVPG correlates with post-transplant hepatic fibrosis (B2).

\section{Assessing hepatic fibrosis in rare cases}

There are rare conditions that lead to hepatic fibrosis and PHT with subsequent variceal bleeding. These include cystic fibrosis (CF), schistosomiasis, congenital hepatic fibrosis, etc. Markers of hepatic fibrosis have not been well utilized in such conditions apart from cases of CF.

\section{Cystic fibrosis}

Liver disease is the third leading cause of mortality in patients with $\mathrm{CF}$ and can result in progressive liver failure and portal hypertension (PHT) in about $30 \%$ of patients. However, detection of CF-associated liver disease (CFLD) is challenging. Many studies have been done in the recent years to assess the role of different non-invasive markers in assessing liver fibrosis and PHT in patients with CF [177-182]. A recent study by Sadler et al. showed that the AUROC curves for FS, APRI and FT for the detection of CFLD were 0.78 (95\% CI 0.65-0.92), 0.72 (95\% CI $0.56-0.87$ ) and 0.76 (95\% CI 0.62-0.90; $p$ not significant).
At a threshold of $>5.2 \mathrm{kPa}$, the sensitivity, specificity, positive and negative predictive values of LSM according to FS for detecting CFLD were 67, 83, 40 and $94 \%$, respectively [177]. Also, LSM was the only variable associated with CFLD (OR 2.74, $95 \%$ CI 1.53-4.89; $p=0.001)$ in a study by Kitson et al. [179]. The AUROC for LSM predicting CFLD was 0.87 (95\% CI 0.77-0.98), and LSM $\geq 6.8 \mathrm{kPa}$ predicted CFLD with $76.0 \%$ sensitivity and $92.0 \%$ specificity. Median LSM was higher in those with PHT [15.7 kPa (IQR 9.2-17.2) versus $5.4 \mathrm{kPa}$ (IQR 4.3-6.8); $p<0.001]$. The AUROC curve for LSM predicting the presence of PHT was 0.96 (95\% CI $0.92-1.00$ ). LSM $\geq 6.8 \mathrm{kPa}$ is highly suggestive of CFLD, and LSM $<8.9 \mathrm{kPa}$ reliably excludes PHT [178]. This indicates that LSM is an accurate and reliable non-invasive tool in assessing CFLD and PHT. A study by Karlas et al. $[179,180]$ utilized the use of TE and ARFI in differentiating between cases of $\mathrm{CF}$ with and without cirrhosis $(n=50)$ and compared them with a control group with alcoholic cirrhosis. Their finding showed that patients with CFLD with liver cirrhosis differed significantly from other CFLD patients (ARFI: 1.49 versus $1.13 \mathrm{~m} / \mathrm{s} ; p=0.031$; TE 7.95 versus $4.16 \mathrm{kPa} ; p=0.020$ ) and had significantly lower results than individuals with alcoholic liver cirrhosis (ARFI 1.49 versus $2.99 \mathrm{~m} / \mathrm{s} ; p=0.002$ ). Pediatric patients $(n=12)$ with CF-related PHT and esophageal varices (EV) had higher FibroScan values than those without varices with median values of $22.4 \mathrm{kPa}(14.4-30.4 \mathrm{kPa})$ versus $7.9 \mathrm{kPa}(4.4-13.7 \mathrm{kPa} ; p=0.01)$. Using a threshold of $12 \mathrm{kPa}$, four of six patients without EV would not have needed endoscopy [181]. Furthermore, TIMP-1 had a high diagnostic accuracy for CFLD in adults. The diagnostic sensitivities were increased when $\mathrm{TE}$ and the respective biomarkers were combined for the detection of CFLD and EV [182].

\section{Schistosomal hepatic periportal fibrosis (SH-PPF)}

Schistosoma infection is a frequent cause of PHT and EV in Middle Eastern countries, Africa, South America and the Far East. In a study by $\mathrm{Wu}$ et al. performed in 55 patients with advanced Schistosomiasis japonica, utilizing LB as a gold-standard, HA and INR were reliable markers for differentiating significant liver fibrosis in patients with advanced Schistosomiasis japonica. The authors concluded that this new simple index can easily predict significant SHF with a high degree of accuracy [183]. The platelet count/spleen diameter ratio has been found useful in predicting the presence of $\mathrm{EV}$ in patients with advanced hepatosplenic schistosomiasis in upper endoscopy [184]. LSM using FibroScan and reference needle-LB was done in 229 patients with $\mathrm{CHC}$; $29 \%$ of them had positive serology for schistosomiasis [185]. In this subgroup, the 
sensitivity of FibroScan in detecting fibrosis stages 2-3 was found to be impaired. However, multivariate logistic regression showed that fibrosis stages (F0-F1 and F4) were the most independent factors that were associated with the agreement between FibroScan and LB (OR 3.4, 7.12 and $p<0.001, p<0.001$, respectively) [185]. The only study that assessed the role of non-invasive markers and TE in the detection of SH-PPF in patients with pure schistosomiasis ( $n=30 ; 28$ of them had splenomegaly and 25 had $\mathrm{EV})$ showed that the mean $\pm \mathrm{SD}$ LSM was $9.4 \pm 5.5 \mathrm{Kpa}$ (range $3.5-30 \mathrm{Kpa}$ ). By TE, F0-F2 was detected in 22 $(73.3 \%)$ cases, and F3-4 was detected in the remaining 8 $(26.7 \%)$. In addition, EV and splenomegaly were detected in $18 / 25(81.8 \%)$ and $19 / 27(86.4 \%)$ patients with nonadvanced fibrosis and in $7(87.5 \%)$ and $8(100 \%)$ patients with advanced fibrosis, respectively $(p=0.71$ and $p=0.27$, respectively) [186]. TE is not useful in the diagnosis of SH-PPF and EV in patients with pure schistosomiasis. However, these findings need to be confirmed in a larger group of patients. Interestingly, Shiha and Zalata have previously shown that the presence of schistosomiasis does not interfere with the application of the Knodell score in patients with CHC [187].

\section{Consensus statements}

- Elastography should be recommended for all CFLDs to assess the stage of fibrosis to differentiate between those with CFLD with and without cirrhosis, to follow its progression and to predict the presence of EV (A1).

- Larger studies are needed to assess for the role of various non-invasive tools in assessing periportal fibrosis in pure cases of schistosomiasis (B1).

- Studying the mechanism(s) underlying the failure of FibroScan to assess fibrosis in cases with pre-hepatic portal hypertension is (are) warranted (A1).

\section{Future prospects}

LB still remains the gold standard for quantifying liver fibrosis. Quantitation of fibrosis by LB remains an inexact science, even with technologies such as morphometric analysis. LB could soon shift from routine light microscopy to digital image analysis by virtual microscopy and incorporation of numerical measurements in conjunction with integrated analysis of cell functions at the DNA, RNA, protein and signaling level.

FibroScan and the currently available serologic tests can make the differentiation between early and advanced disease, which means that they could be used to guide treatment decisions. However, in the future we need proven applicable methods, which are able to monitor the dynamic nature of fibrosis progression and regression. This will require serum markers that are sufficiently sensitive to measure small changes in the state of the ECM. Unfortunately, none of the current non-invasive methods have this degree of sensitivity. It is also important to investigate the prognostic value of non-invasive methods of fibrosis detection.

Continued progress can be anticipated in unraveling the molecular regulation of fibrosis and its treatment. Rapid advances in gene therapy, tissue-specific targeting and high-throughput small molecule screening of cytokine inhibitors are likely to benefit the diagnosis of hepatic fibrosis.

New insights into the regulation of growth, apoptosis and intracellular signaling by cytokines, adipokines and hormones could have direct implications for stellate cell behavior in liver injury. Accelerating progress is certain once methods of non-invasive diagnosis are established that enable rapid assessment of fibrosis in clinical trials, and ultimately in clinical practice

\section{References}

1. Shiha G, Sarin SK, Ibrahim AE, Omata M, Kumar A, Lesmana LA, et al. Liver fibrosis: consensus recommendations of the Asian Pacific Association for the Study of the Liver (APASL). Hepatol Int 2009;3:323-333

2. Guyatt GH, Oxman AD, Vist GE, Kunz R, Falck-Ytter Y, Alonso-Coello P, et al. GRADE: an emerging consensus on rating quality of evidence and strength of recommendations. BMJ 2008;336:924-926

3. Schünemann HJ, Oxman AD, Brozek J, Glasziou P, Jaeschke R, Vist GE, et al. Grading quality of evidence and strength of recommendations for diagnostic tests and strategies. BMJ 2008;336:1106-1110

4. Mulherin SA, Miller WC. Spectrum bias or spectrum effect? Subgroup variation in diagnostic test evaluation. Ann Intern Med 2002;137:598-602

5. Poynard T, Ngo Y, Perazzo H, Munteanu M, Lebray P, Moussalli J, et al. Prognostic value of liver fibrosis biomarkers: a meta-analysis. Gastroenterol Hepatol (NY) 2011;7:445-454

6. Guha IN, Rosenberg WM. Noninvasive assessment of liver fibrosis: serum markers, imaging, and other modalities. Clin Liver Dis 2008;12:883-900.

7. Goehring C, Goehring C1, Perrier A, Morabia A. Spectrum bias: a quantitative and graphical analysis of the variability of medical diagnostic test performance. Stat Med 2004;23:125-135

8. Poynard T, Halfon P, Castera L, Munteanu M, Imbert-Bismut F, Ratziu V, et al. Standardization of ROC curve areas for diagnostic evaluation of liver fibrosis markers based on prevalences of fibrosis stages. Clin chem 2007;53:1615-1622

9. Menghini G. One-second needle biopsy of the liver. Gastroenterology 1958;35:190-199

10. Grigorescu M. Noninvasive biochemical markers of liver fibrosis. J Gastrointestin Liver Dis 2006;15:149-159 
11. Scott DA, Netchvolodoff CV, Bacon BR. Delayed subcapsular hematoma after percutaneous liver biopsy is a manifestation of warfarin toxicity. Am J Gastroenterol 1991;86:503-505

12. Rockey DC, Caldwell SH, Goodman ZD, Nelson RC, Smith $\mathrm{AD}$, American Association for the Study of Liver Diseases. Liver biopsy. Hepatology 2009;49:1017-1044

13. Judmaier G, Prior C, Klimpfinger M, Bernklau E, Vogel W, Dietze $\mathrm{O}$, et al. Is percutaneous liver biopsy using the 'Tru-Cut' '(Travenol) needle superior to Menghini puncture? Z Gastroenterol 1989;27:657-661

14. Colombo M, Del Ninno E, de Franchis R, De Fazio C, Festorazzi S, Ronchi G, et al. Ultrasound assisted percutaneous liver biopsy: superiority of the Tru-Cut over the Menghini needle for diagnosis of cirrhosis. Gastroenterology 1988;95:487-489

15. Lindor KD, Bru C, Jorgensen RA, Rakela J, Bordas JM, Gross $\mathrm{JB}$, et al. The role of ultrasonography and automatic-needle biopsy in outpatient percutaneous liver biopsy. Hepatology 1996;23:1079-1083

16. McGill DB, Rakela J, Zinsmeister AR, Ott BJ. A 21-year experience with major hemorrhage after percutaneous liver biopsy. Gastroenterology 1990;99:1396-1400

17. Piccinino F, Sagnelli E, Pasquale G, Giusti G. Complications following percutaneous liver biopsy. A oumadiner retrospective study on 68,276 biopsies. J Hepatol 1986;2:165-173

18. Tobin MV, Gilmore IT. Plugged liver biopsy in patients with impaired coagulation. Dig Dis Sci 1989;34:13-15

19. Bialecki ES, Ezenekwe AM, Brunt EM, Collins BT, Ponder TB, Bieneman BK, et al. Comparison of liver biopsy and noninvasive methods for diagnosis of hepatocellular carcinoma. Clin Gastroenterol Hepatol 2006;4:361-368

20. Tung WC, Huang YJ, Leung SW, Kuo FY, Tung HD, Wang JH, et al. Incidence of needle tract seeding and responses of soft tissue metastasis by hepatocellular carcinoma post-radiotherapy. Liver Int 2007;27:192-200

21. Khuroo MS, Wani NA, Javid G, Khan BA, Yattoo GN, Shah $\mathrm{AH}$, et al. Percutaneous drainage compared with surgery for hepatic hydatid cysts. N Engl J Med 1997;337:881-887

22. Ewe K. Bleeding after liver biopsy does not correlate with indices of peripheral coagulation. Dig Dis Sci 1981;26:388-393

23. Trotter JF, Olson J, Lefkowitz J, Smith AD, Arjal R, Kenison J. Changes in international normalised ratio (INR) and model for end-stage liver disease (MELD) based on selection of clinical laboratory. Am J Transpl 2007;7:1624-1628

24. Caldwell SH, Hoffman M, Lisman T, Macik BG, Northup PG, Reddy KR, et al. Coagulation disorders and hemostasis in liver disease: pathophysiology and critical assessment of current management. Hepatology 2006;44:1039-1046

25. Abdel-Wahab OI, Healy B, Dzik WH. Effect of fresh-frozen plasma transfusion on prothrombin time and bleeding in patients with mild coagulation abnormalities. Transfusion 2006;46:1279-1285

26. Tripodi A, Chantarangkul V, Primignani M, Fabris F, Dell'Era A, Sei C, et al. The international normalised ratio calibrated for cirrhosis [INR- (liver)] normalizes prothrombin time results for model for end-stage liver disease calculation. Hepatology 2007;46:520-527

27. Saxena P, Bihari C, Rastogi A, Agarwal S, Anand L, Sarin SK. Sonoclot signature analysis in patients with liver disease and its correlation with conventional coagulation studies. Adv Hematol 2013;2013:237351

28. Kim ES. Lusutrombopag: first global approval. Drugs 2016;76:155-158

29. van Leeuwen DJ, Wilson L, Crowe DR. Liver biopsy in the mid1990s: questions and answers. Semin Liver Dis $1995 ; 15: 340-359$
30. Janes $\mathrm{CH}$, Lindor KD. Outcome of patients hospitalized for complications after outpatient liver biopsy. Ann Intern Med 1993;118:96-98

31. Van Thiel DH, Gavaler JS, Wright H, Tzakis A. Liver biopsy. Its safety and complications as seen at a liver transplant center. Transplantation 1993;55:1087-1090

32. Kalambokis G, Manousou P, Vibhakorn S, Marelli L, Cholongitas E, Senzolo M, et al. Transjugular liver biopsy: indications, adequacy, quality of specimens, and complications: a systematic review. J Hepatol 2007;47:284-294

33. Miraglia R, Luca A, Gruttadauria S, Minervini MI, Vizzini G, Arcadipane A, et al. Contribution of transjugular liver biopsy in patients with the clinical presentation of acute liver failure. Cardiovasc Intervent Radiol 2006;29:1008-1010

34. Azoulay D, Raccuia JS, Roche B, Reynes M, Bismuth H. The value of early transjugular liver biopsy after liver transplantation. Transplantation 1996;61:406-409

35. Behrens G, Ferral H. Transjugular liver biopsy. Semin Intervent Radiol 2012;29:111-117

36. Beckmann MG, Bahr MJ, Hadem J, Bredt M, Wedemeyer H, Schneider AS, et al. Clinical relevance of transjugular liver biopsy in comparison with Percutaneous and laparoscopic liver biopsy. Gastroenterol Res Pract 2009;94701428

37. Ble M, Procopet B, Miquel R, Hernandez-Gea V, García-Pagán JC. Transjugular liver biopsy. Clin Liver Dis 2014;18:767-778

38. Li FQ, Ko GY, Sung KB, Gwon DI, Ko HK, Kim JW, et al. Transfemoral liver biopsy using a Quick-Core biopsy needle system in living donor liver transplantation recipients. Liver Transpl 2014;20:1178-1184

39. Clark TW, McCann JW, Salsamendi J, Dowd MF, Hajdu CH. Optimizing needle direction during transjugular liver biopsy provides superior biopsy specimens. Cardiovasc Intervent Radiol 2014;37:1540-1545

40. Denzer U, Arnoldy A, Kanzler S, Galle PR, Dienes HP, Lohse AW. Prospective randomized comparison of mini-laparoscopy and Percutaneous liver biopsy: diagnosis of cirrhosis and complications. J Clin Gastroenterol 2007;41:103-110

41. Steele K, Schweitzer MA, Lyn-Sue J, Kantsevoy SV. Flexible transgastric peritoneoscopy and liver biopsy: a feasibility study in human beings (with videos). Gastrointest Endosc 2008;68:61-66

42. The Royal College of Pathologists. Tissue pathways for liver biopsies for the investigation of medical disease and for focal lesions. The Royal College of Pathologists, 2008

43. Bedossa P. Sampling variability of liver fibrosis in chronic hepatitis C. Hepatology 2003;38:1449-1457

44. Colloredo G1, Guido M, Sonzogni A, Leandro G. Impact of liver biopsy size on histological evaluation of chronic viral hepatitis: the smaller the sample, the milder the disease. J Hepatol 2003;39:239-244

45. Cholongitas E, Senzolo M, Standish R, Marelli L, Quaglia A, Patch D, et al. A systematic review of the quality of liver biopsy specimens. Am J Clin Pathol 2006;125:710-721

46. Cholongitas E, Quaglia A, Samonakis D, Senzolo M, Triantos C, Patch D, et al. Transjugular liver biopsy: how good is it for accurate histological interpretation? Gut 2006;55:1789-1794

47. Riley TR 3rd, Ruggiero FM. The effect of processing on liver biopsy core size. Dig Dis Sci 2008;53:2775-2777

48. Bull HJ, Gilmore IT, Bradley RD, Marigold JH, Thompson RP. Experience with transjugular liver biopsy. Gut 1983;24:1057-1060

49. Hall AR, Tsochatzis E, Morris R, Burroughs AK, Dhillon AP. Sample size requirement for digital image analysis of collagen proportionate area in cirrhotic livers. Histopathology 2013;62:421-430 
50. Scheuer PJ. Liver biopsy size matters in chronic hepatitis: bigger is better. Hepatology 2003;38:1356-1358

51. Guido M, Mangia A, Faa G; Gruppo Italiano Patologi Apparato Digerente (GIPAD); Società Italiana di Anatomia Patologica e Citopatologia Diagnostica/International Academy of Pathology, Italian division (SIAPEC/IAP). Chronic viral hepatitis: the histology report. Dig Liver Dis 2011;43(Suppl 4):S331-S343

52. De Groote J, Desmet VJ, Gedigk P, Korb G, Popper H, Poulsen $\mathrm{H}$, et al. A classification of chronic hepatitis. Lancet 1968;2:626-628

53. Knodell RG, Ishak KG, Black WC, Chen TS, Craig R, Kaplowitz N, et al. Formulation and application of a numerical scoring system for assessing histological activity in asymptomatic chronic active hepatitis. Hepatology 1981;1:431-435

54. Ishak K, Baptista A, Bianchi L, Callea F, De Groote J, Gudat F, et al. Histological grading and staging of chronic hepatitis. J Hepatol 1995;22:696-699

55. Bedossa P, Poynard T. An algorithm for the grading of activity in chronic hepatitis C. The METAVIR Cooperative Study Group. Hepatology 1996;24:289-293

56. Standish RA, Cholongitas E, Dhillon A, Burroughs AK, Dhillon AP. An appraisal of the histopathological assessment of liver fibrosis. Gut 2006;55: 569-578

57. Rousselet MC, Michalak S, Dupré F, Croué A, Bedossa P, SaintAndré JP, et al. Sources of variability in histological scoring of chronic viral hepatitis. Hepatology 2005;41:257-264

58. Gailhouste L, Le Grand Y, Odin C, Guyader D, Turlin B, Ezan $\mathrm{F}$, et al. Fibrillar collagen scoring by second harmonic microscopy: a new tool in the assessment of liver fibrosis. J Hepatol 2010;52:398-406

59. Goodman ZD, Stoddard AM, Bonkovsky HL, Fontana RJ, Ghany MG, Morgan TR, et al. Fibrosis progression in chronic hepatitis C: morphometric image analysis in the HALT-C trial. Hepatology 2009;50:1738-1749

60. O'Brien MJ1, Keating NM, Elderiny S, Cerda S, Keaveny AP, Afdhal $\mathrm{NH}$, et al. An assessment of digital image analysis to measure fibrosis in liver biopsy specimens of patients with chronic hepatitis C. Am J Clin Pathol 2000;114:712-718

61. Maduli E, Andorno S, Rigamonti C, Capelli F, Morelli S, Colombi S, et al. Evaluation of liver fibrosis in chronic hepatitis $\mathrm{C}$ with a computer-assisted morphometric method. Ann Ital Med Int 2002;17:242-247

62. Pilette C, Rousselet MC, Bedossa P, Chappard D, Oberti F, Rifflet $\mathrm{H}$, et al. Histopathological evaluation of liver fibrosis: quantitative image analysis vs semi-quantitative scores. J Hepatol 1998;28:439-446

63. Xu S, Wang Y, Tai DC, Wang S, Cheng CL, Peng Q, et al. qFibrosis: a fully-quantitative innovative method incorporating histological features to facilitate accurate fibrosis scoring in animal model and chronic hepatitis B patients. J Hepatol 2014;61:260-269

64. Isse K, Grama K, Abbott IM, Lesniak A, Lunz JG, Lee WM, et al. Adding value to liver (and allograft) biopsy evaluation using a combination of multiplex quantum dot immunostaining, high-resolution whole-slide digital imaging, and automated image analysis. Clin Liver Dis 2010;14:669-685

65. Molleken C, Mölleken C, Sitek B, Henkel C, Poschmann G, Sipos B, et al. Detection of novel biomarkers of liver cirrhosis by proteomic analysis. Hepatology 2009;49:1257-1266

66. Werling K, Szentirmay Z, Szepesi A, Schaff Z, Szalay F, Szabó $Z$, et al. Hepatocyte proliferation and cell cycle phase fractions in chronic viral hepatitis $\mathrm{C}$ by image analysis method. Eur $\mathrm{J}$ Gastroenterol Hepatol 2010;13:489-493

67. Shiha GE, Zalata KR, Abdalla AF, Mohamed MK. Immunohistochemical identification of HCV target antigen in paraffin- embedded liver tissue: reproducibility and staining patterns. Liver Int 2005;25:254-260

68. Khalid SS, Hamid S, Siddiqui AA, Qureshi A, Qureshi N. Gene profiling of early and advanced liver disease in chronic hepatitis C patients. Hepatol Int 2011;5:782-788

69. Furuta K, Sato S, Yamauchi T, Ozawa T, Harada M, Kakumu S. Intrahepatic gene expression profiles in chronic hepatitis B and autoimmune liver disease. J Gastroenterol 2008;43:866-874

70. Mas V1, Maluf D, Archer KJ, Potter A, Suh J, Gehrau R, et al. Transcriptome at the time of hepatitis $\mathrm{C}$ virus recurrence may predict the severity of fibrosis progression after liver transplantation. Liver Transpl 2011;17:824-835

71. Tripodo C, Petta S, Guarnotta C, Pipitone R, Cabibi D, Colombo MP, et al. Liver follicular helper T-cells predict the achievement of virological response following interferon-based treatment in HCV-infected patients. Antivir Ther 2012;17:111-118

72. Xu R, Zhang Z, Wang FS. Liver fibrosis: mechanisms of immune-mediated liver injury. Cell Mol Immunol 2012;9:296-301

73. Rojkind, Giambrone MA, Biempica L. Collagen types in normal and cirrhotic liver. Gastroenterology 1979;76:710-719

74. Gressner AM, Krull N, Bachem MG. Bachem, Regulation of proteoglycan expression in fibrotic liver and cultured fat storing cells. Pathol Res Pract 1994;190:864-882

75. Arthur MJ. Fibrogenesis II. Metalloproteinases and their inhibitors in liver fibrosis, Am J Physiol Gastrointest Liver Physiol 2000;279:G245-G249

76. Gressner OA, Weiskirchen R, Gressner AM. Biomarkers of liver fibrosis: clinical translation of molecular pathogenesis or based on liver-dependent malfunction tests, Clin Chim Acta 2007;38:107-113

77. Veidal SS, Bay-Jensen AC, Tougas G, Karsdal MA, Vainer B. Serum markers of liver fibrosis: combining the BIPED classification and the neo-epitope approach in the development of new biomarkers. Dis Markers 2010;28:15-28

78. Hayasaka A, Koch J, Schuppan D, Maddrey WC, Hahn EG. The serum concentrations of the aminoterminal propeptide of procollagen type III and the hepatic content of mRNA for the alpha 1 chain of procollagen type III in carbon tetrachloride-induced rat liver fibrogenesis. J Hepatol 1991;13:328-338

79. Xie Q, Zhou X, Huang P, Wei J, Wang W, Zheng S. The performance of enhanced liver fibrosis (ELF) test for the staging of liver fibrosis: ameta-analysis. PLoS One 2014;9:e92772

80. Innes HA, McDonald SA, Dillon JF, Allen S, Hayes PC, Goldberg D, et al. Towards a more complete understanding of the association between a hepatitis $\mathrm{C}$ sustained viral response and cause-specific outcomes. Hepatology 2015;62:355-364

81. Tamaki N, Kurosaki M, Kuno A, Korenaga M, Togayachi A, Gotoh $\mathrm{M}$, et al. Wisteria floribunda agglutinin positive human Mac-2-binding protein as a predictor of hepatocellular carcinoma development in chronic hepatitis $\mathrm{C}$ patients. Hepatol Res 2015;45:E82-E88

82. Tamaki N, Kurosaki M, Izumi N. Noninvasive estimation of liver fibrosis and hepatocellular carcinoma development using WFA(+)-M2BP in chronic hepatitis C patients. Rinsho Byori 2015;63:1039-1043

83. Poynard $\mathrm{T}$, Morra R, Ingiliz $\mathrm{P}$, Imbert-Bismut $\mathrm{F}$, Thabut $\mathrm{D}$, Messous D, et al. Biomarkers of liver fibrosis. Adv Clin chem 2008;46:131-160

84. Udell JA, Wang CS, Tinmouth J, FitzGerald JM, Ayas NT, Simel DL, et al. Does this patient with liver disease have cirrhosis? JAMA 2012;307:832-842

85. Fontana RJ, Lok AS. Noninvasive monitoring of patients with chronic hepatitis C. Hepatology 2002;36(5 Suppl. 1):S57-S64 
86. Afzal S, Masroor I, Beg M. Evaluation of chronic liver disease: does ultrasound scoring criteria help? Int $\mathrm{J}$ Chronic Dis 2013;2013:326231

87. Wilson SR, Withers CE. The Liver. In Rumack CM, Wilson SR, Charboneau JW, Johnson JAM (eds). Diagnostic Ultrasound, 3rd Edition. Philadelphia: Mosby, 2005

88. Giorgio A, Amoroso P, Lettieri G, Fico P, de Stefano G, Finelli L, et al. Cirrhosis: value of caudate to right lobe ratio in diagnosis with ultrasound. Radiology 1986;161:443-445

89. Taylor KJ, Riely CA, Hammers L, Flax S, Weltin G, GarciaTsao G, et al. Quantitative ultrasound attenuation in normal liver and in patients with diffuse liver disease. Importance of fat. Radiology 1986;160:65-71

90. Murakami T, Kuroda C, Marukawa T, Harada K, Wakasa K, Sakurai M, et al. Regenerating nodules in hepatic cirrhosis. MR findings with pathologic correlation. AJR Am J Rontgen 1990;155:1227-1231

91. Shen L, Li JQ, Zeng MD, Lu LG, Fan ST, Bao H. Correlation between ultrasonographic and pathologic diagnosis of liver fibrosis due to chronic virus hepatitis. World J Gastroenterol 2006;12:1292-1295

92. Choong CC, Venkatesh SK, Siew EP. Accuracy of routine clinical ultrasound for staging of liver fibrosis. J Clin Imaging Sci 2012;2:58

93. Berzigotti A, Piscaglia F, EFSUMB Education and Professional Standards Committee. Ultrasound in portal hypertension-part 2and EFSUMB recommendations for the performance and reporting of ultrasound Correlation between ultrasonographic and pathologic diagnosis of liver fibrosis due to chronic virus hepatitis examinations in portal hypertension. Ultraschall Med 2012;33:8-32

94. Bouakaz A, de Jong N. WFUMB Safety symposium on echocontrast agents: nature and types of ultrasound contrast agents. Ultrasound Med Biol 2007;33:187-196

95. Maruyama H, Ishibashi H, Takahashi M, Imazeki F, Yokosuka $\mathrm{O}$. Effect of signal intensity from the accumulated microbubbles in the liver for differentiation of idiopathic portal hypertension from liver cirrhosis. Radiology 2009;252:587-594

96. Fritz GA, Schoellnast H, Deutschmann HA, Wiltgen M, Brader $\mathrm{P}$, Berghold A, et al. Density histogram analysis of unenhanced hepatic computed tomography in patients with diffuse liver diseases. J Comput Assist Tomogr 2006;30:201-205

97. Colagrande S, Centi N, Galdiero R, Ragozzino A. Transient hepatic intensity differences: part 2 , those not associated with focal lesions. AJM Am J Roentgenol 2007;188:160-166

98. Van Beers BE, Leconte I, Materne R, Smith AM, Jamart J, Horsmans Y. Hepatic perfusion parameters in chronic liver disease: dynamic CT measurements correlated with disease severity. AJM Am J Roentgenol 2001;176:667-673

99. Kapanen M, Halavaara J, Häkkinen AM. Comparison of liver perfusion parameters studied with conventional extravascular and experimental intravascular CT contrast agents. Acad Radiol 2007; 14:951-958

100. Kudo M, Zheng RQ, Kim SR, Okabe Y, Osaki Y, Iijima H, et al. Diagnostic accuracy of imaging for liver cirrhosis compared to histologically proven liver cirrhosis. A multicenter collaborative study. Intervirology 2008;51(Suppl 1):17-26

101. Huber A, Ebner L, Montani M, Semmo N, Roy Choudhury K, Heverhagen $\mathrm{J}$, et al. Computed tomography findings in liver fibrosis and cirrhosis. Swiss Med Wkly 2014;144:w13923

102. Talwalkar JA, Yin M, Fidler JL, Sanderson SO, Kamath PS, Ehman RL. Magnetic resonance imaging of hepatic fibrosis: emerging clinical applications. Hepatology 2008;47:332-342

103. Aguirre DA, Behling CA, Alpert E, Hassanein TI, Sirlin CB. Liver fibrosis: noninvasive diagnosis with double contrast material-enhanced MR imaging. Radiology 2006;239:425-437
104. Wang QB, Zhu H, Liu HL, Zhang B. Performance of magnetic resonance elastography and diffusion-weighted imaging for the staging of hepatic fibrosis: A meta-analysis. Hepatology 2012;56:239-247

105. Wong VW, Chu WC, Wong GL, Chan RS, Chim AM, Ong A, et al. Prevalence of non-alcoholic fatty liver disease and advanced fibrosis in Hong Kong Chinese: a population study using proton-magnetic resonance spectroscopy and transient elastography. Gut 2012;61:409-415

106. Ziol M, Handra-Luca A, Kettaneh A, Christidis C, Mal F, Kazemi F, et al. Noninvasive assessment of liver fibrosis by measurement of stiffness in patients with chronic hepatitis $\mathrm{C}$. Hepatology 2005;41:48-54

107. Talwalkar JA, Kurtz DM, Schoenleber SJ, West CP, Montori VM. Ultrasound-based transient elastography for the detection of hepatic fibrosis: systematic review and meta-analysis. Clin Gastroenterol Hepatol 2007;5:1214-1220

108. Friedrich-Rust M, Ong MF, Martens S, Sarrazin C, Bojunga J, Zeuzem S, et al. Performance of transient elastography for the staging of liver fibrosis: a meta-analysis. Gastroenterology 2008;134:960-974

109. Adebajo CO, Talwalkar JA, Poterucha JJ, Kim WR, Charlton MR. Ultrasound-based transient elastography for the detection of hepatic fibrosis in patients with recurrent hepatitis $\mathrm{C}$ virus after liver transplantation: a systematic review and meta-analysis. Liver Transpl 2012;18:323-331

110. Millonig G, Friedrich S, Adolf S, Fonouni H, Golriz M, Mehrabi A, et al. Liver stiffness is directly influenced by central venous pressure. J Hepatol 2010;52:206-210

111. Castera L, Foucher J, Bernard PH, Carvalho F, Allaix D, Merrouche W, et al. Pitfalls of liver stiffness measurement: a 5-year prospective study of 13,369 examinations. Hepatology 2010;5:828-835

112. Myers RP, Pomier-Layrargues G, Kirsch R, Pollett A, Beaton M, Levstik M, et al. Discordance in fibrosis staging between liver biopsy and transient elastography using the FibroScan XL probe. J Hepatol 2012;56:564-270

113. de Lédinghen V, Wong VW, Vergniol J, Wong GL, Foucher J, $\mathrm{Chu} \mathrm{SH}$, et al. Diagnosis of liver fibrosis and cirrhosis using liver stiffness measurement: comparison between M and XL probe of FibroScan ${ }^{\circledR}$. J Hepatol 2012;56:833-839

114. Wong VW, Vergniol J, Wong GL, Foucher J, Chan AW, Chermak F, et al. Liver stiffness measurement using XL probe in patients with nonalcoholic fatty liver disease. Am J Gastroenterol 2012;107:1862-1871

115. Tapper EB, Cohen EB, Patel K, Bacon B, Gordon S, Lawitz E, et al. Levels of alanine aminotransferase confound use of transient elastography to diagnose fibrosis in patients with chronic hepatitis C virus infection. Clin Gastroenterol Hepatol 2012;10:932-937

116. Roulot D, Czernichow S, Le Clésiau H, Costes JL, Vergnaud $\mathrm{AC}$, Beaugrand $\mathrm{M}$, et al. Liver stiffness values in apparently healthy subjects: influence of gender and metabolic syndrome. J Hepatol 2008;48:606-613

117. Gaia S, Carenzi S, Barilli AL, Bugianesi E, Smedile A, Brunello $F$, et al. Reliability of transient elastography for the detection of fibrosis in non-alcoholic fatty liver disease and chronic viral hepatitis. J Hepatol 2011;54:64-71

118. Mueller S, Millonig G, Sarovska L, Friedrich S, Reimann FM, Pritsch M, et al. Increased liver stiffness in alcoholic liver disease: differentiating fibrosis from steatohepatitis. World J Gastroenterol 2010;16:966-972

119. Ochi H, Hirooka M, Koizumi Y, Miyake T, Tokumoto Y, Soga $\mathrm{Y}$, et al. Real-time tissue elastography for evaluation of hepatic fibrosis and portal hypertension in nonalcoholic fatty liver diseases. Hepatology 2012;56:1271-1278 
120. Singh S, Fujii LL, Murad MH, Wang Z, Asrani SK, Ehman RL, et al. Liver stiffness is associated with risk of decompensation, liver cancer, and death in patients with chronic liver diseases: a systematic review and meta-analysis. Clin Gastroenterol Hepatol 2013;11:1573-1584

121. Cescon M, Colecchia A, Cucchetti A, Peri E, Montrone L, Ercolani G, et al. Value of transient elastography measured with FibroScan in predicting the outcome of hepatic resection for hepatocellular carcinoma. Ann Surg 2012;256:706-712

122. Nguyen-Khac E, Nahon P, Boursier J, Moreno C, Starkel P, Mueller S, et al. Non-invasive diagnosis of liver fibrosis by fibroscan in patients with alcoholic liver disease: a meta-analysis with individual data. J Hepatol 2014;60(Suppl. 1):S34

123. Corpechot C, Gaouar A, El Naggar A, Kemgang D, Wendum R, Poupon F, et al. Liver stiffness assessed by fibroscan is a major prognostic factor in primary sclerosing cholangitis. J Hepatol 2014;60(Suppl. 1):S34

124. Friedrich-Rust M, Nierhoff J, Lupsor M, Sporea I, FierbinteanuBraticevici C, Strobel D, et al. Performance of acoustic radiation force impulse imaging for the staging of liver fibrosis: a pooled meta-analysis. J Viral Hepat 2012;19:e212-219

125. Nierhoff J, Chávez Ortiz AA, Herrmann E, Zeuzem S, FriedrichRust M. The efficiency of acoustic radiation force impulse imaging for the staging of liver fibrosis: a meta-analysis. Eur Radiol 2013;23:3040-3053

126. Yap WW, Kirke R, Yoshida EM, Owen D, Harris AC. Noninvasive assessment of liver fibrosis using ARFI with pathological correlation, a prospective study. Ann Hepatol 2013;12:608-615

127. Bota S, Sporea I, Sirli R, Popescu A, Gradinaru-Tascau O. How useful are ARFI elastography cut-off values proposed by metaanalysis for predicting the significant fibrosis and compensated liver cirrhosis? Med Ultrason 2015;17:200-205

128. Liu H, Fu J, Hong R, Liu L, Li F. Acoustic radiation force impulse elastography for the non-invasive evaluation of hepatic fibrosis in non-alcoholic fatty liver disease patients: A systematic review \& meta-analysis. PLoS One. 2015;10:e0127782

129. Bota S, Herkner H, Sporea I, Salzl P, Sirli R, Neghina AM, et al. Meta-analysis: ARFI elastography versus transient elastography for theevaluation of liver fibrosis. Liver Int 2013;33:1138-1147

130. Kobayashi K, Nakao H, Nishiyama T, Lin Y, Kikuchi S, Kobayashi Y, et al. Diagnostic accuracy of real-time tissue elastography for the staging of liver fibrosis: a meta-analysis. Eur Radiol 2015;25:230-238

131. Muller M, Gennisson JL, Deffieux T, Tanter M, Fink M. Quantitative viscoelasticity mapping of human liver using supersonic shear imaging: preliminary in vivo feasibility study. Ultrasound Med Biol 2009;35:219-229

132. Sirli R, Bota S, Sporea I, Jurchis A, Popescu A, GradinaruTascău $\mathrm{O}$, et al. Liver stiffness measurements by means of supersonic shear imaging in patients without known liver pathology. Ultrasound Med Biol 2013;39:1362-137

133. Pinzani M, Pinzani M, Vizzutti F, Arena U, Marra F. Technology Insight: noninvasive assessment of liver fibrosis by biochemical scores and elastography. Nat Clin Pract Gastroenterol Hepatol 2008;5:95-106

134. Yoneda M, Thomas E, Sclair SN, Grant TT, Schiff ER. Supersonic shear imaging and transient elastography with the $\mathrm{XL}$ probe accurately detect fibrosis in overweight or obese patients with chronic liver disease. Clin Gastroenterol Hepatol 2015;13:1502-1509

135. Jiang Y, Li GY, Qian LX, Hu XD, Liu D, Liang S, et al. Characterization of the nonlinear elastic properties of soft tissues using the supersonic shear imaging (SSI) technique: inverse method, ex vivo and in vivo experiments. Med Image Anal 2015;20:97-111
136. Deffieux T, Gennisson JL, Bousquet L, Corouge M, Cosconea S, Amroun $\mathrm{D}$ et al. Investigating liver stiffness and viscosity for fibrosis, steatosis and activity staging using shear wave elastography. J Hepatol 2015;62:317-324

137. Sporea I, Bota S, Jurchis A, Sirli R, Grădinaru-Tascău O, Popescu A, et al. Acoustic radiation force impulse and supersonic shear imaging versus transient elastography for liver fibrosis assessment. Ultrasound Med Biol 201;39:1933-1941

138. Cassinotto C, Boursier J, de Lédinghen V, Lebigot J, Lapuyade $\mathrm{B}$, Cales $\mathrm{P}$, et al. Liver stiffness in nonalcoholic fatty liver disease: a comparison of supersonic shear imaging, FibroScan, and ARFI with liver biopsy. Hepatology 2016;63:1817-1827

139. Bavu E, Gennisson JL, Couade M, Bercoff J, Mallet V, Fink M, et al. Noninvasive in vivo liver fibrosis evaluation using supersonic shear imaging: a clinical study on 113 hepatitis $\mathrm{C}$ virus patients. Ultrasound Med Biol 2011;37:1361-1373

140. Cassinotto C, Lapuyade B, Mouries A, Hiriart JB, Vergniol J, Gaye D, et al. Noninvasive assessment of liver fibrosis with impulse elastography: comparison of supersonic shear imaging with ARFI and FibroScan ${ }^{\circledR}$. J Hepatol 2014;61:550-557

141. Venkatesh SK, Venkatesh SK, Yin M, Ehman RL. Magnetic resonance elastography of liver: technique, analysis, and clinical applications. J Magn Reson Imaging 2013;37:544-555

142. Low G, Kruse SA, Lomas DJ. General review of magnetic resonance elastography. World J Radiol 2016;8:59-72

143. Huwart L, Sempoux C, Vicaut E, Salameh N, Annet L, Danse E, et al. Magnetic resonance elastography for the noninvasive staging of liver fibrosis. Gastroenterology 2008;135:32-40

144. Venkatesh SK, Wang G, Lim SG, Wee A. Magnetic resonance elastography for the detection and staging of liver fibrosis in chronic hepatitis B. Eur Radiol 2014;24:70-78

145. Guo Y, Parthasarathy S, Goyal P, McCarthy RJ, Larson AC, Miller FH. Magnetic resonance elastography and acoustic radiation force impulse for staging hepatic fibrosis: a meta-analysis. Abdom Imaging 2015;40:818-834

146. Ichikawa S, Motosugi U, Ichikawa T, Sano K, Morisaka H, Enomoto N, et al. Magnetic resonance elastography for staging liver fibrosis in chronic hepatitis C. Magn Reson Med Sci 2012;11:291-297

147. Chen J, Talwalkar JA, Yin M, Glaser KJ, Sanderson SO, Ehman RL et al. Early detection of nonalcoholic steatohepatitis in patients with nonalcoholic fatty liver disease by using MR elastography. Radiology 2011;259:749-756

148. Kim D, Kim WR, Talwalkar JA, Kim HJ, Ehman RL. Advanced fibrosis in nonalcoholic fatty liver disease: noninvasive assessment with MR elastography. Radiology 2013;268:411-419

149. Bensamoun SF, Leclerc GE, Debernard L, Cheng X, Robert L, Charleux F, et al. Cutoff values for alcoholic liver fibrosis using magnetic resonance elastography technique. Alcoholism, clinical and experimental research 2013;37:811-817

150. Russo MW, Galanko JA, Zacks SL, Beavers KL, Fried MW, Shrestha R. Impact of donor age and year of transplant on graft survival in liver transplant recipients with chronic hepatitis C. Am J Transplant 2004;4:1133-1138

151. Toniutto P, Fabris C, Avellini C, Minisini R, Bitetto D, Rossi E, et al. Excess body weight, liver steatosis, and early fibrosis progression due to hepatitis $\mathrm{C}$ recurrence after liver transplantation. World J Gastroenterol 2005; 11:5944-5950

152. Lee SH, Joo DJ, Kim SU, Kim MS, Lee AL, Choi GH, et al. Graft function measured by transient elastography in living donor liver transplantation: preliminary. Transplant Proc 2013;45:3028-3031

153. Howell JAngus P, Gow P. Hepatitis C recurrence: the Achilles heel of liver transplantation. Transpl Infect Dis 2014;16:1-16

154. Carlson JJ, Kowdley KV, Sullivan SD, Ramsey SD, Veenstra DL. An evaluation of the potential cost-effectiveness of non- 
invasive testing strategies in the diagnosis of significant liver fibrosis. J Gastroenterol Hepatol 2009;24:786-791

155. Agency for Drugs and Techniques in Health (CADTH). Diagnosis and monitoring of liver fibrosis in patients with chronic hepatitis C: a review of the clinical evidence and cost effectiveness. Rapid response report. Summary and Critical Appraisal March 8, 2012

156. Liu S, Schwarzinger M, Carrat F, Goldhaber-Fiebert JD. Cost effectiveness of fibrosis assessment prior to treatment for chronic hepatitis C patients. PLoS One 2011;6(12):e26783

157. García-Jurado L, Oyagüez I, Casado MÁ, Tural C, GonzálezGarcía J, Ortega E, et al. Evaluation of the costs of transient elastography (FibroScan $\left({ }^{\circledR}\right)$ ) in the diagnosis of liver fibrosis in HIV patients with hepatitis C virus. Enferm Infec Microbiol Clin 2012;30:294-299

158. Stevenson M, Lloyd-Jones M, Morgan MY, Wong R. Non-invasive diagnostic assessment tools for the detection of liver fibrosis in patients with suspected alcohol-related liver disease: a systematic review and economic evaluation. Health Technology Assessment 2012;16:1-174

159. Canavan C, Eisenburg J, Meng L, Corey K, Hur C. Ultrasound elastography for fibrosis surveillance is cost effective in patients with chronic hepatitis $\mathrm{C}$ virus in the UK. Dig Dis Sci 2013;58:2691-2704

160. Ripoll C, Groszmann R, Garcia-Tsao G, Grace N, Burroughs A, Planas R, et al. Hepatic venous pressure gradient predicts clinical decompensation in patients with compensated cirrhosis. Gastroenterology 2007;133:481-488

161. Pomier-Layrargues G, Kusielewicz D, Willems B, Villeneuve JP, Marleau D, Côté J, et al. Presinusoidal portal hypertension in nonalcoholic cirrhosis. Hepatology 1985;5:415-418

162. Rector Jr WG. Propranolol for portal hypertension. Evaluation of therapeutic response by direct measurement of portal vein pressure. Arch Intern Med 1985;145:648-650

163. Valla D, Bercoff E, Menu Y, Bataille C, Lebrec D. Discrepancy between wedged hepatic venous pressure and portal venous pressure after acute propranolol administration in patients with alcoholic cirrhosis. Gastroenterology 1984;86:1400-1403

164. Perello A, Escorsell A, Bru C, Gilabert R, Moitinho E, GarcíaPagán JC, et al. Wedged hepatic venous pressure adequately reflects portal pressure in hepatitis $\mathrm{C}$ virus-related cirrhosis. Hepatology 1999;30:1393-1397

165. Deplano A, Migaleddu V, Pischedda A, Garrucciu G, Gregu G, Multinu $\mathrm{C}$, et al. Portohepatic gradient and portal hemodynamics in patients with cirrhosis due to hepatitis $\mathrm{C}$ virus infection. Dig Dis Sci 1999;44:155-162

166. Okuda K, Okuda K, Suzuki K, Musha H, Arimuzu N. Percutaneous transhepatic catheterization of the portal vein for the study of portal hemodynamics and shunts: A preliminary report. Gastroenterology 1977;73:279-284

167. Keiding S, Vilstrup H. Intrahepatic heterogeneity of hepatic venous pressure gradient in human cirrhosis again. Scand J Gastroenterol 2002;37:1344

168. Thalheimer U, Leandro G, Samonakis DN, Triantos CK, Patch $\mathrm{D}$, Burroughs AK. Assessment of the agreement between wedge hepatic vein pressure and portal vein pressure in cirrhotic patients. Dig Liver Dis 2005;37:601-608

169. Thalheimer U, Mela M, Patch D, Burroughs AK. Monitoring target reduction in hepatic venous pressure gradient during pharmacological therapy of portal hypertension: a close look at the evidence. Gut 2004;53:143-148

170. Guha IN, Myers RP, Patel K, Talwalkar JA. Biomarkers of liver fibrosis: what lies beneath the receiver operating characteristic curve? Hepatology 2011;54:1454-1462

171. Suk KT, Kim DJ, Kim CH, Park SH, Cheong JY, Cho SW, et al. Diagnostic accuracy of hepatic venous pressure gradient measurement in the prediction of stage 1 compensated liver cirrhosis in patients with chronic hepatitis B. Eur J Gastroenterol Hepatol 2013;25:1170-1176

172. Kumar M, Kumar A, Hissar S, Jain P, Rastogi A, Kumar D, et al. Hepatic venous pressure gradient as a predictor of fibrosis in chronic liver disease because of hepatitis B virus. Liver Int 2008;28:690-698

173. Suk KT, Kim DJ, Kim CH, Park SH, Cheong JY, Cho SW, et al. Diagnostic accuracy of biomarkers measured in the hepatic vein and peripheral vein in the prediction of advanced fibrosis in patients with chronic viral hepatitis. Clin Biochem 2012;45:1075-1080

174. Vizzutti F, Arena U, Romanelli RG, Rega L, Foschi M, Colagrande $\mathrm{S}$, et al. Liver stiffness measurement predicts severe portal hypertension in patients with HCV-related cirrhosis. Hepatology 2007;45:1290-1297

175. Bureau C, Metivier S, Peron JM, Selves J, Robic MA, Gourraud PA, et al. Transient elastography accurately predicts presence of significant portal hypertension in patients with chronic liver disease. Aliment Pharmacol Ther 2008;27:1261-1268

176. Carrión JA, Navasa M, Bosch J, Bruguera M, Gilabert R, Forns $\mathrm{X}$. Transient elastography for diagnosis of advanced fibrosis and portal hypertension in patients with hepatitis $\mathrm{C}$ recurrence after liver transplantation. Liver Transpl 2006;12:1791-1798

177. Sadler MD, Crotty P, Fatovich L, Wilson S, Rabin HR, Myers RP. Noninvasive methods, including transient elastography, for the detection of liver disease in adults with cystic fibrosis. Can J Gastroenterol Hepatol 2015;29:139-144

178. Karlas T, Neuschulz M, Oltmanns A, Wirtz H, Keim V, Wiegand J. ARFI and transient elastography for characterization of cystic fibrosis related liver disease: first longitudinal follow-up data in adult patients. J Cyst Fibros 2013;12:826-827

179. Kitson MT, Kemp WW, Iser DM, Paul E, Wilson JW, Roberts SK. Utility of transient elastography in the non-invasive evaluation of cystic fibrosis liver disease. Liver Int 2013;33:698-705

180. Karlas T, Neuschulz M, Oltmanns A, Güttler A, Petroff D, Wirtz $\mathrm{H}$, et al. Non-invasive evaluation of cystic fibrosis related liver disease in adults with ARFI, transient elastography and different fibrosis scores. PLoS One 2012;7:e42139

181. Rath T, Menendez KM, Kügler M, Hage L, Wenzel C, Schulz R, et al. TIMP-1/-2 and transient elastography allow non invasive diagnosis of cystic fibrosis associated liver disease. Dig Liver Dis 2012;44:780-787

182. Malbrunot-Wagner AC, Bridoux L, Nousbaum JB, Riou C, Dirou A, Ginies JL, et al. Transient elastography and portal hypertension in pediatric patients with cystic fibrosis Transient elastography and cystic fibrosis. J Cyst Fibros 2011;10:338-342

183. Wu YM, Xu N, Hu JY, Xu XF, Wu WX, Gao SX, et al. A simple noninvasive index to predict significant liver fibrosis in patients with advanced schistosomiasis japonica. Parasitol Int 2013;62:283-288

184. Agha A, Abdulhadi MM, Marenco S, Bella A, Alsaudi D, ElHaddad A, et al. Use of the platelet count/spleen diameter ratio for the noninvasive diagnosis of esophageal varices in patients with schistosomiasis. Saudi J Gastroenterol 2011;17:307-311

185. Esmat G, Elsharkawy A, El Akel W, Fouad A, Helal K, Mohamed MK, et al. Fibroscan of chronic HCV patients coinfected with schistosomiasis. Arab J Gastroenterol 2013; 14:109-112

186. Shiha G, Samir W, Soliman R, Elbasiony M, Ahmed N, Helmy A. Transient Elastography (FibroScan) in not useful in the diagnosis of schistosomal hepatic peri-portal fibrosis. (accepted as poster presentation in APASL meeting, Japan, 2016)

187. Shiha G, Zalata KR. Does schistosomiasis interfere with application of Knodell score for assessment of chronic hepatitis C? Med Sci Monit 2001;7 Suppl 1:241-245 